Preprints of the

Max Planck Institute for

Research on Collective Goods

Bonn 2013/20

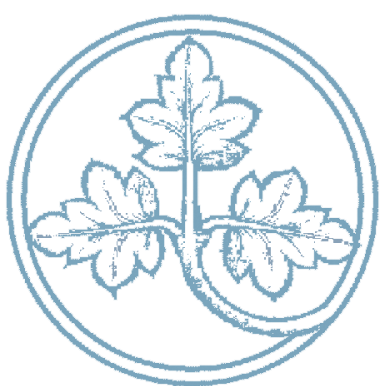

Eckpunkte für einen stärker ökonomisch informierten Ansatz im Recht der nichtsteuerlichen Abgaben

Stefan Magen

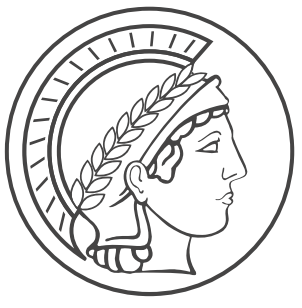




\title{
Eckpunkte für einen stärker ökonomisch informierten Ansatz im Recht der nicht-steuerlichen Abgaben
}

\author{
Stefan Magen
}

October 2013 


\section{Einleitung}

Anders als Private kann der Staat seinen Finanzbedarf durch Zwangsabgaben einseitig auf Kosten der Bürger decken, ohne sich den Zwängen des Marktes aussetzen zu müssen ${ }^{1}$. Finanzverfassungsrecht und Finanzwissenschaften (die mit öffentlichen Finanzen befasste Disziplin der Volkswirtschaftslehre) teilen die Einschätzung, dass eine solche Befugnis der Begrenzung und Einhegung durch rechtliche Regeln bedarf ${ }^{2}$. Beide Disziplinen tendieren allerdings zu unterschiedlichen Schlussfolgerungen in der Frage, was aus dieser Sorge für das Verhältnis von Steuern und nicht-steuerlichen Abgaben folgt. Aus Sicht des Finanzverfassungsrechts sollte sich der Staat im Grundsatz aus Steuern finanzieren (sog. Steuerstaatsprinzip) ${ }^{3}$. Nicht-steuerliche Abgaben sollten demgegenüber die Ausnahme bleiben. Die finanzverfassungsrechtlichen Regelungen des Grundgesetzes über die Steuern entfalteten gegenüber diesen, so das BVerfG, zugleich eine „Begrenzungs- und Schutzfunktion“, weshalb die Erhebung nicht-steuerlicher Abgaben durch das Erfordernis eines besonderen sachlichen Rechtfertigungsgrundes ,grundlegend begrenzt" werde ${ }^{4}$. In den Finanzwissenschaften trifft man eher auf die gegenläufige Forderung, die Steuerfinanzierung staatlicher Aufgaben wo möglich durch eine Gebührenfinanzierung zu ersetzen ${ }^{5}$. Abgaben nach dem Äquivalenzprinzip an staatliche Gegenleistungen zu knüpfen erscheint als probates Mittel, um maßlose Steuererhebung und verschwenderische Ausgabenpolitik zu bändigen ${ }^{6}$. Denn so werden Abgabe und Ausgabe aneinander gebunden und beide finden ihr Maß am Nutzen für den Bürger. Mit dieser Stoßrichtung ist die ökonomische Analyse nicht-steuerlicher Abgaben denn auch im Finanzverfassungsrecht hervorgetreten: „Vom Steuerstaat zum Gebührenstaat“67 .

Ein grundsätzlicher Systemwechsel steht derzeit allerdings nicht auf der Tagesordnung ${ }^{8}$. Rechtspraxis und Rechtswissenschaft sind vielmehr damit beschäftigt, die rechtlichen Grenzen für die Zulässigkeit, Ausgestaltung und Anwendung bestimmter Typen nicht-steuerlicher Abgaben und konkreter Abgabentatbestände auszubuchstabieren. Die Finanzwissenschaften

1 Das schließt indirekte Zwänge nicht aus, vgl. zur Gebührenerhebung Gawel, Die Verwaltung 44 (2011), S. 327 (328 f.).

$2 \quad$ P. Kirchhof, in: Isensee/ders. (Hrsg.), HStR V, 3. Aufl. 2007, § 118 Rn. 6 ff.; Waldhoff, in: Ebd., § 116 Rn. 3. Brennan/Buchanan, The Power to Tax, 1980.

3 Vgl. BVerfGE 78, 249 (267); 93, 319 (342); Heun, in: Sacksofsky/Wieland (Hrsg.), Vom Steuerstaat zum Gebührenstaat, 2000, S. $10 \mathrm{ff}$.

$4 \quad$ BVerfGE 122, 316 (333 ff.); 123, 132 (140 f.); 124, 235 (243); 124, 348 (364 ff.); BVerfG, NJW 2013, S. $638 \mathrm{f}$. Der Grund ist, eine Umgehung der finanzverfassungsrechtlichen Gesetzgebungskompetenzen und Verteilungsregeln für Steuern zu verhindern, den Bürger vor weiteren Zugriffen auf seine Ressourcen zu schützen und der Belastungsgleichheit der Abgabenpflichten Rechnung zu tragen.

5 Grossekelter, in: Sacksofsky/Wieland (Hrsg.), Vom Steuerstaat zum Gebührenstaat (FN 3), S. 24 ff.; Hansjürgens, Äquivalenzprinzip und Staatsfinanzierung, 2001; vgl. auch Schmehl, Das Äquivalenzprinzip im Recht der Staatsfinanzen, 2004. Grundlegend zum Äquivalenzprinzip Wicksell, Finanztheoretische Untersuchungen, 1896, S. 76 ff.

6 Zur Maßlosigkeit der Steuererhebung aus finanzverfassungsrechtlicher Sicht $P$. Kirchhof, in: HStR V (FN 2), § 118 Rn. 14.

7 So der Titel des einflussreichen Sammelbands von Sacksofsky/Wieland (Hrsg.), Vom Steuerstaat zum Gebührenstaat, 2000; zur Kritik vgl. etwa P. Kirchhof, in: HStR V (FN 2), § 119 Rn. 3 ff.

8 Für einen solchen Systemwechsel wäre das Steuerstaatsprinzip im Übrigen nicht die einzige und vielleicht auch nicht die gravierendste finanzverfassungsrechtliche Hürde. Schwierigere Probleme wirft es auf, dass die erhoffte Disziplinierung der Staatsausgaben durch einen gebührenfinanzierten Staat nur dann wirksam wäre, wenn man zugleich die staatlichen Steuererhebungskompetenzen drastisch einschränkt. 
analysieren nicht-steuerliche Abgaben aber, wie man sehen wird, nicht von den Abgabenarten her, sondern setzen bei den Staatsaufgaben an, für die eine Finanzierung über nichtsteuerliche Abgaben in Betracht kommt: bei dem Bau und Unterhalt öffentlicher Straßen, der Bereitstellung kommunaler Schwimmbäder oder Friedhöfe, der öffentlichen Abfallentsorgung, dem öffentlicher Rundfunk, usw. Aus ökonomischer Sicht könnte also zum Beispiel gefragt werden, wie es zu bewerten ist, wenn Straßen privat oder staatlich gebaut und betrieben werden, wenn öffentliche Straßen über Steuern, eine öffentlich-rechtliche Maut oder vertragliche Entgelte finanziert werden, wenn die öffentlich-rechtliche Maut dabei als Gebühr, Beitrag oder Sonderabgabe gefasst ist, wie die Mauttarife ausgestaltet und bemessen sein sollten, und nicht zuletzt wie sich die gewählte Bereitstellung und Finanzierungsform auf private Konkurrenten auswirkt und umgekehrt ${ }^{9}$. Weil die ökonomische Analyse nicht-steuerlicher Abgaben bei der konkret finanzierten Staatsaufgabe ansetzt, gelangt man zu greifbaren ökonomischen Aussagen zu einzelnen Abgabentatbeständen in der Regel erst, wenn alle relevanten Wirkzusammenhänge in den Blick genommen und theoretisch begründete Aussagen auf belastbare Empirie gestützt werden. Das ist aber nicht das Ziel der folgenden Ausführungen, die auf einzelne Abgabentatbestände nur zur Veranschaulichung eingehen. Beabsichtigt ist hier ein strukturierter Überblick mit dem Ziel, Eckpunkte der ökonomischen Analyse von nicht-steuerlichen Abgaben herauszuarbeiten, und zwar so, wie sie aus rechtswissenschaftlicher Sicht anschlussfähig und aussagekräftig erscheinen ${ }^{10}$. Diese Eckpunkte mögen zu einer stärker ökonomisch informierten Behandlung abgabenrechtlicher Fragestellungen anregen und sollen Anknüpfungspunkte zu den Finanzwissenschaften aufzeigen. Intensiven Kontakt zur Ökonomie gibt es hier bislang vor allem mit der Betriebswirtschaftslehre über Fragen der Kostenkalkulation bei Gebühren sowie zur Umweltökonomie über Fragen der Lenkungssteuern. Für die Rechtswissenschaften wäre es ein Rationalitätsgewinn, diesen Austausch in die Tiefe und in der Breite fortzusetzen - insbesondere über die Struktur der finanzverfassungsrechtlichen Rechtfertigung und zu den diversen Bereichen der Daseinsvorsorge - um sich bei den Finanzwissenschaften über die Wirkungs- und Belastungszusammenhänge zwischen spezifischen Typen von Staatsaufgaben und hoheitlichen Finanzierungsinstrumenten informieren zu lassen. Ausgehend von einer Deutung von Staatsaufgaben als staatliche Produktion von Gütern und Dienstleistungen bietet die finanzverfassungsrechtliche Rechtfertigungslehre für nicht-steuerliche Abgaben eine probate Verbindung mit der finanzwissenschaftlichen Kollektivgütertheorie (unten II.). Im Anschluss werden einige Aspekte der Rolle nicht-steuerlicher Abgaben aus Sicht der Kollektivgütertheorie angerissen, und zwar zunächst für die staatliche Produktion kollektiver Güter (unten III.) und dann für deren Nutzung (unten IV.).

9 Zur finanzwissenschaftlichen Analyse des Fernstraßenbaus Gawel/Schmidt, Finanzwissenschaftliche Probleme der Gebührenfinanzierung von Verkehrsinfrastruktur nach dem Fernstraßengesetz, 2010.

10 Nicht immer geht das ohne Abstriche an der auf unterschiedliche Weise in den Rechtswissenschaften wie in den Wirtschaftswissenschaften gepflegten Präzision. 


\section{Nicht-steuerliche Abgaben, Staatsaufgaben und kollektive Güter}

\section{Der Staat als Produzent kollektiver Güter und Dienstleistungen}

Zugespitzt lässt sich das Verhältnis von Staatsrecht und Finanzwissenschaften begreifen als gegenläufige Betrachtung desselben Gegenstandes „Staat“: Das öffentliche Recht erfasst den Staat als Ausübung von Staatsgewalt mit dem Zweck der Erfüllung öffentlicher Aufgaben und regelt deren demokratische Legitimation und rechtsstaatliche Bindung. Dass der Staat dafür Ressourcen in Anspruch nimmt, die er durch Einnahmen finanzieren muss, ist als regelungsbedürftiger Tatbestand anerkannt, steht aber nicht im Mittelpunkt des Interesses. Die finanzwissenschaftliche Betrachtungsweise erfasst den Staat umgekehrt als eine Institution, die knappe Ressourcen in Anspruch nimmt, um Güter (unter Einschluss von Dienstleistungen) zu produzieren ${ }^{11}$. Dass der Staat dabei Zwangsgewalt in Anspruch nimmt, die legitimiert und geregelt werden muss, ist als Mittel kollektiver Güterproduktion anerkannt, aber nur von instrumenteller Bedeutung für die effiziente (und vielleicht auch verteilungsgerechte) Nutzung knapper Ressourcen ${ }^{12}$.

Nicht aus Freiheits-, sondern aus Effizienzgründen sollte der Staat sich dabei auf die Produktion solcher Güter beschränken, die der Markt weniger effizient bereitstellen würde ${ }^{13}$. Er sollte deshalb nicht private, sondern kollektive Güter produzieren (zur Terminologie später), und diese auch nicht auf Märkten gegen Entgelte tauschen, sondern über Steuern und andere Abgaben finanzieren. „Produktion“ meint in den Finanzwissenschaften also nicht Produktion für den Markt. Entsprechend wird der Staat als Produzent kollektiver Güter auch nicht als gewinnmaximierendes Unternehmen vorgestellt, sondern als Institution, die kollektive Güter ausschließlich im Interesse der Bürger produzieren sollte - idealerweise als ,wohlwollender Diktator“, realistischerweise aber eher als eigennütziger,,Agent“, den die Bürger als „Prinzipale“ nur unvollkommen kontrollieren können ${ }^{14}$. Den Finanzwissenschaften liegt auch ein weiter Begriff des Gutes zugrunde, der alles umfasst, was der Bedürfnisbefriedigung dient, insbesondere auch ideelle Güter und Dienstleistungen ${ }^{15}$. Auch der Rechtsstaat bzw. die Bereitstellung einer funktionsfähigen Rechtspflege ist demnach ein Gut im finanzwissenschaftlichen Sinn, das unter Aufwendung staatlicher Mittel produziert wird. Zum Beispiel sah der Haushalt des Landes NRW im Jahr 2011 ca. 3,5 Mrd. € an Ausgaben für den Bereich des Justizministeriums vor, denen ca. 1 Mrd. $€$ an Einnahmen aus Gebühren gegenüberstanden (da-

11 Musgrave/Musgrave, Public Finance, 5. Aufl., 1989; Blankart, Öffentliche Finanzen in der Demokratie, 8. Aufl., 2011, S. 9; Brümmerhoff, Finanzwissenschaften, 10. Aufl. 2011, S. 10 ff.; Fritsch, Marktversagen und Wirtschaftspolitik, 8. Aufl. 2011, S. 325; Zimmermann/Henke/Broer, Finanzwissenschaft, 11. Aufl. 2012, S. 3 ff.

12 Nach der bekannten Lehre von Musgrave bestehen die drei Funktionen des Staates in Allokation, Distribution und (makro-ökonomischer) Stabilisierung; vgl. Musgrave/Musgrave (FN 11), S. 3 ff.

13 Fritsch, (FN 11).

$14 \mathrm{Zu}$ diesen beiden Schulen vgl. Buchanan/Musgrave, Public Finance and Public Choice. Two Contrasting Views of the State, 1999.

15 Vgl. Brümmerhoff, (FN 11), S. 19; zu Dienstleistungen in der Ökonomie siehe Korte, in: Callies/Korte, Dienstleistungsrecht in der EU, 2011, § 2 . 
von ca. 0,95 Mrd. $€$ aus der ordentlichen Gerichtsbarkeit) ${ }^{16}$. Aus finanzwissenschaftlicher Sicht wäre es nun absurd, würde man die Differenz von ca. 2,5 Mrd. € zwischen Gebühreneinnahmen und Kosten als volkswirtschaftlichen Verlust ansehen und die Arbeit der Justiz deshalb als ineffizient beklagen. Denn der Nutzen, der die Ausgaben des Justizressorts finanzwissenschaftlich rechtfertigt, liegt nicht in den Gebühreneinnahmen, sondern in den mannigfaltigen, häufig indirekten, zum großen Teil auch nicht-monetären und in ihrer Gesamtheit kaum zu messenden positiven Wirkungen, die ein funktionierendes Rechtssystem für die Bürger einer Gesellschaft hat ${ }^{17}$. Sie werden besser durch Steuern, und nicht durch Gebühren finanziert.

\section{Die ökonomischen Eigenschaften von Gütern als finanzwissenschaftliche „Staatsaufgabenlehre“}

Während die finanzwissenschaftliche Analyse von Steuern in der Regel von der Mittelverwendung und damit von den Staatsaufgaben abstrahiert, werden nicht-steuerliche Abgaben im Zusammenhang der Bewältigung bestimmter Marktversagensprobleme thematisiert ${ }^{18}$. Vereinfacht gesagt wird der Grund für Marktversagen in der nicht-marktvermittelten Beeinflussung des Nutzens anderer Bürger gesehen (positive oder negative Externalitäten) und insbesondere auf die ökonomischen Charakteristika bestimmter Klassen von Gütern zurückgeführt (die man auch als Spezialfälle von Externalitätenproblemen betrachten kann) ${ }^{19}$. In dem durch die Erhebung nicht-steuerlicher Abgaben abgegrenzten Gegenstandsbereich analysieren die Finanzwissenschaften also die Erfüllung von Staatsaufgaben (unter anderem) als Probleme der Produktion kollektiver Güter, der Bewältigung von positiven und negativen Externalitäten und ggf. der Umsetzung von Verteilungszwecken ${ }^{20}$.

Verschiedene Arten von kollektiven Gütern (öffentliche Güter, Klubgüter und Gemeingüter) und von Externalitätenproblemen (positive und negative) werfen allerdings unterschiedliche

16 Vgl. Haushaltsplan für den Geschäftsbereich des Justizministeriums für das Haushaltsjahr 2011, erhältlich unter www.fm.fin-nrw.de.

17 Zur zentralen Bedeutung eines funktionierenden Rechtssystems für den wirtschaftlichen Wohlstand jüngst Acemoglu/Robinson, Why Nations Fail: The Origins of Power, Prosperity, and Poverty, 2012; Cooter/Schäfer, Solomon's Knot: How Law Can End the Poverty of Nations, 2013.

18 Zum Markt als „Referenzsystem“ Fritsch, (FN 11), S. 6 ff.; Zimmermann/Henke/Broer, (FN 11), S. 49 ff.

19 Samuelson, Review of Economics and Statistics 36 (1954), S. 387 ff., Cornes/Sandler, The Theory of Externalities, Public Goods and Club Goods, 2. Aufl. 1996; Fritsch, (FN 11), S. 80 ff.; zu den Unterschieden Münch, Kollektive Güter und Gebührenelemente einer Gebührentheorie für Kollektivgüter, 1976, S. $36 \mathrm{ff}$.

20 Arnold, Theorie der Kollektivgüter, 1992; Brümmerhoff, (FN 11) S. 56 ff.; Cornes/Sandler, (FN 19); Fritsch, (FN 11), S. 80 ff.; Grossekettler, in Sacksofsky/Wieland (Hrsg.), (FN 3), S. 24 ff.; ders., Vahlens Kompendium der Wirtschaftstheorie und Wirtschaftspolitik, Bd. 1, 9. Aufl. 2007, S. 575 ff.; Hindrik/Myles, Intermediate Public Economics, 2. Aufl. 2013; Mueller, Public Choice III, 2003, S. 9 ff.; Musgrave/Musgrave, Public Finance in Theory and Practice, 5. Aufl. 1989, S. 41 ff.; Musgrave, Public Finance, in: Durlauf/Blume (Hrsg.), The New Palgrave Dictionary of Economics, 2. Aufl. 2008; Nowotny/Zagler, Der öffentliche Sektor, 5. Aufl., 2009, S. 60 ff.; Zimmermann/Henke/Broer (FN 11), S. 49 ff.; zum Stand der Forschung Hellwig, in: Max Planck Institute for Research on Collective Goods (Hrsg.), Status Report 2009-2011, S. 17 ff. (erhältlich unter www.coll.mpg.de); grundlegend etwa Lindahl, Die Gerechtigkeit der Besteuerung, 1919; Samuelson (FN 19); Musgrave, The Theory of Public Finance, 1959; Buchanan, Economica 32 (1965), S. 1 ff.; Clarke, Public Choice 11 (1971), S. 17 ff. 
Probleme auf (s. unten 4), für die aus finanzwissenschaftlicher Sicht unterschiedliche staatliche Interventionen als Lösungen in Betracht kommen. Nicht alle Lösungen involvieren dabei den Einsatz nicht-steuerlicher Abgaben, sondern auch Entgelte, Steuern oder ordnungsrechtliche Lösungen können u.a. in Betracht kommen. Ein Thema der ökonomischen Analyse ist deshalb, die relativen Vor- und Nachteile verschiedener Lösungen zu vergleichen. Entscheidet sich der Staat für nicht-steuerliche Abgaben, müssen diese je nach Art des kollektiven Gutes bzw. Externalitätenproblems unterschiedliche Funktionen erfüllen. Ein zentrales Thema für die ökonomische Analyse nicht-steuerlicher Abgaben ist deshalb die Frage, wie Abgaben so ausgestaltet werden können, dass sie ökonomisch sinnvoll funktionieren. Die finanzwissenschaftliche Analyse von nicht-steuerlichen Abgaben ist also (1) eine Analyse konkreter Kollektivguts- und Externalitätenprobleme, für die (2) neben zweckgebundenen Abgaben u.a. auch Lösungen über Entgelte, Steuern oder Ordnungsrecht in Betracht zu ziehen sind, wobei (3) zweckgebundene Abgaben auf das zugrundeliegende ökonomische Problem zugeschnitten sein sollten ${ }^{21}$. Sie ist damit ,in der Regel eine Korrespondenzbetrachtung zwischen Gutscharakteristik der bereitgestellten Leistungen und hierzu adäquaten Finanzierungsinstrumen$\operatorname{ten}^{\text {‘622 }}$.

Man kann sich dies an zwei Beispielen verdeutlichen: Frequenzzuteilungen nach $\S 55$ TKG dienen u.a. dazu, eine störungsfreie Nutzung des Frequenzspektrums sicherzustellen ( 2 Abs. $2 \mathrm{Nr} .7 \mathrm{TKG})^{23}$, und lassen sich deshalb finanzwissenschaftlich als Regelung zur Begrenzung negativer Externalitäten bzw. für die Nutzung eines Gemeingutes verstehen. Es geht also um den Umgang mit Knappheit bei der Nutzung eines Gutes, die der Markt mangels privater Verfügungsrechte an Frequenzen nicht bewältigt ${ }^{24}$. Für viele Frequenznutzungen wählt das Telekommunikationsrecht insoweit eine ordnungsrechtliche Herangehensweise, bei der nur Verwaltungsgebühren erhoben werden. Diese Aufgabe bewältigt auch eine Versteigerung von Mobilfunklizenzen. Die höhere Gebühr, über die dann die Erlöse aus der Versteigerung erhoben werden, bekommt dann allerdings den Charakter einer Verleihungsgebühr ${ }^{25}$, deren rechtfertigender Sachzweck hier in einer Vorteilsabschöpfung ${ }^{26}$ oder in dem Lenkungszweck einer

21 Am Beispiel der öffentlichen Interessen im Gebührenrecht: Gawel, Verwaltungsarchiv 102 (2011), S. 491 ff.

22 Gawel, Die kommunalen Gebühren, 1995, S. 26 (Hervorhebungen dort).

23 Säcker, in: ders. (Hrsg.), Telekommunikationsgesetz, 3. Aufl. 2013, § 2 Rn. 13.

24 Die nach $\S 62$ TKG mögliche Übertragung öffentlich-rechtlicher Frequenzzuteilungen (dazu Clausi, Rechtsfragen des Frequenzhandels, 2004) führt dagegen nicht zu privaten Verfügungsrechten, sondern zu handelbaren öffentlich-rechtlichen Rechten (zum Unterschied Magen, Handelbare öffentliche Rechte, in: Kirchhof/Korte/ders. (Hrsg.), Öffentliches Wettbewerbsrecht, im Erscheinen).

25 Kämmerer, NVwZ 2002, S. 161 (168); Schumacher, NJW 2000, S. 3096 (3098 ff.); Becker, Die Verwaltung 35 (2002), S. 1 (17 ff.); zur Kategorie der Verleihungsgebühr Murswiek, NUR 1994, S. 170; Heimlich, Die Verleihungsgebühr als Umweltabgabe, 1996; ders., DÖV 1997, S. 996 ff.

26 Kritisch Becker, (FN 25), S. 1 (17 ff.); bejahend Wollenschläger, Verteilungsverfahren, 2010, S. 420 ff.; ausführlich Martini, Der Markt als Instrument hoheitlicher Verteilungslenkung, 2008, S. 512 ff. Zur Zulässigkeit der Vorteilsabschöpfung als Gebührenzweck BVerfGE 93, 319 (345), 108, 1 (18); BVerfG, NVwZ 2013, S. 638 ff.; aus der juristischen Diskussion Meyer, Gebühren für die Nutzung von Umweltressourcen, 1995; Murswiek, NVwZ 1996, S. 417 ff.; Raber, NVwZ 1997, S. 219 ff.; Sacksofsky, Umweltschutz durch nicht-steuerliche Abgaben, 2000; aus finanzwissenschaftlicher Sicht Gawel, Umweltabgaben zwischen Steuer- und Gebührenlösung, 1999; ders. et al., Weiterentwicklung von Abwasserabgabe und Wasserentnahmeentgelten zu einer umfassenden Wassernutzungsabgabe, 2011. 
effizienten Nutzung des Frequenzspektrums liegen kann (vgl. § 2 Abs. 2 Nr. 7 TKG) ${ }^{27}$. Eine ökonomische Analyse der genauen Effizienz- und Verteilungswirkungen kann insoweit eine rationalisierende und einnahmenbeschränkende Wirkung haben, weil sie es erlaubt, die Geeignetheit der Allokationsmechanismen und die Plausibilität der zur Rechtfertigung der Gebührenerhebung vorgebrachten Sachzwecke zu überprüfen und so hilft, verdeckte Fiskalinteressen aufzudecken ${ }^{28}$. Rundfunkbeiträge dagegen werden zur Finanzierung eines Gutes erhoben, dessen Nutzung gerade nicht von Knappheit geprägt ist, weil die einmal produzierte und ausgestrahlte Rundfunksendung im Prinzip von beliebig vielen Nutzern empfangen werden kann. Finanzwissenschaftlich handelt es sich um ein öffentliches Gut mit meritorischen Nutzendimensionen (bzw. wegen der mittlerweile verfügbaren Verschlüsselungstechnologien um ein Klubgut, das wegen seiner meritorischen Nutzenanteile ohne Zugangsbeschränkung bereitgestellt wird ${ }^{29}$. Die ökonomische Funktion der Rundfunkbeiträge liegt aber nicht darin, wie bei der Frequenzregelung, die Nutzung einer Ressource zu beschränken, um eine Übernutzung zu verhindern, sondern im Gegenteil darin, eine Finanzierungform zu finden, die möglichst wenig vom Gebrauch des gerade unbeschränkt nutzbaren Gutes abhält. Abhängig vom Gutscharakter verlangt Effizienz im einen Fall mithin eine Finanzierung, die die Nutzung auf das wünschenswerte Maß beschränkt, während Abgaben im anderen Fall die Nutzung möglichst wenig beschränken sollten.

\section{Finanzverfassungsrechtliche Rechtfertigung und finanzwissenschaftliche Kollektivgütertheorie}

a) Zum Verhältnis von verfassungsrechtlicher Rechtfertigung und ökonomischer Äquivalenz. Finanzverfassungsrechtlich bedürfen nicht-steuerliche Abgaben wegen der Begrenzungs- und Schutzfunktion der Verfassung einer besonderen sachlichen Rechtfertigung, die nicht schon in dem allgemeinen Fiskalzweck der Generierung staatlicher Einnahmen liegen kann ${ }^{30}$. Das Rechtfertigungserfordernis leitet auch die Abgrenzung von Steuern und nicht-steuerlichen Abgaben. Im Unterschied zu Steuern dürfen nicht-steuerliche Abgaben nicht „voraussetzungslos“ zur Deckung des allgemeinen Finanzbedarfs erhoben und ohne Zweckbindung in den allgemeinen Staatshaushalt eingestellt werden. Vielmehr müssen sie einen Sachzweck verfolgen, der über die bloße Mittelbeschaffung hinausgeht ${ }^{31}$. Es dürfte sich schon angedeutet

27 Martini, (FN 26), S. 582 ff.; Zur effizienten Frequenzallokation aus ökonomischer Sicht Felder, Frequenzallokation in der Telekommunikation, 2004, S. $138 \mathrm{ff}$.

28 Z.B. sind die überhöhten Erlöse aus der UMTS-Auktion wohl auch auf Fehler im gewählten Auktionsdesign zurückzuführen, bei welchem die Auswirkungen des strategischen Verhaltens der Bieter im Bezug auf die Marktstruktur auf den nachgelagerten Märkten nicht richtig eingeschätzt wurden; vgl. Ewerhart/Moldovanu, The German UMTS Design, CESifo Working Paper 680 (9), 2002; Niemeier, Die deutsche UMTS-Auktion, 2002, S. 208 ff. Eine rechtliche Verpflichtung, das Auktionsdesign entsprechend auszugestalten, konstatiert Wollenschläger, (FN. 26), S. 423 u. 427.

29 Vgl. Terschüren, Die Reform der Rundfunkfinanzierung in Deutschland, 2013, S. 194 ff.

30 Reimer/Waldhoff et al., Verfassungsrechtliche Vorgaben für Sonderabgaben des Banken- und Versicherungssektors, 2011, S. 59 f. Das gilt für alle nicht-steuerlichen Abgaben, nicht nur die Sonderabgaben, vgl. BVerfGE 108, 1 (17).

31 BVerfGE 101, 141 (147); 113, 128 (149 f.); 122, 316 (334 f.); 123, 132 (142); P. Kirchhof, in: HStR V (FN 2), § 119 Rn. 79 f.; Waldhoff, in: HStR V (FN 2), § 116 Rn. 91. 
haben, dass die finanzwissenschaftliche Analyse nicht-steuerlicher Abgaben vor allem eine Analyse dieser Sachzwecke ist, genauer gesagt, eine Analyse der Wechselwirkungen zwischen Sachzwecken und Abgabenerhebung.

Nun kommt aus verfassungsrechtlicher Sicht im Prinzip jedes zulässige Gemeinwohlziel als Rechtfertigungsgrund in Betracht. Die einschlägigen finanzverfassungsrechtlichen Vorgaben $^{32}$ führen aber zu einer strukturellen Vorauswahl unter den in Betracht kommenden Gemeinwohlzielen. Das Finanzverfassungsrecht lässt dabei der Tendenz nach nur solche Gemeinwohlziele zu, die einer Äquivalenzbetrachtung zugänglich sind (im finanzverfassungsrechtlichen Sinn, der viel weiter gefasst ist als der gebührenrechtliche Äquivalenzbegriff). Für Gebühren und Beiträge geschieht dies über deren begriffliche Definition als Abgabe für eine individualisierbare staatliche Gegenleistung ${ }^{33}$ bzw. als Abgabe für die potentielle Inanspruchnahme einer staatlichen Gegenleistung ${ }^{34}$. Für die Sonderabgaben sind es die finanzverfassungsrechtlichen Rechtfertigungsvoraussetzungen ${ }^{35}$ die eine Vorstrukturierung in die gleiche Richtung einer finanzwissenschaftlichen Äquivalenzbetrachtung bewirken. Allerdings ist die Gegenseitigkeitsbeziehung hier auf Gruppen statt auf Individuen bezogen und die Verknüpfung von Abgabe und Gegenleistung wesentlich lockerer gefasst. Deshalb liegt bei Sonderabgaben grundsätzlich kein Gegenseitigkeitsverhältnis in dem engen, individualisierten Verständnis vor, das schon aus sich heraus die Abgabe rechtfertigt ${ }^{36}$. Abstrahiert, gelockert und über den Begriff der „Sachnähe“ verschleiert wird der Äquivalenzgedanke aber als GruppenÄquivalenz $z^{37}$ mitgeführt. Die Erträge aus der Sonderausgabe müssen nämlich nachweisbar zugunsten der Gruppe der Abgabenschuldner verwendet werden, die aufgrund einer geteilten Finanzierungsverantwortung eine homogene Gruppe bilden ${ }^{38}$. In der Sache verweist die Finanzierungsverantwortung dabei auf nichts anderes als die materiell bestehenden ökonomischen Wechselbeziehungen ${ }^{39}$.

\section{b) Zum Verhältnis der rechtlich zulässigen Sachzwecke zu den ökonomischen Gütercharakte-} ristika. Hinsichtlich der zulässigen Sachzwecke unterschiedet das Finanzverfassungsrecht ${ }^{40}$ weiter zwischen Finanzierungszwecken, bei denen der Sachzweck in der Verwendung des Aufkommens für eine bestimmte Aufgabe liegt (z.B. der Finanzierung von Studienangeboten an staatlichen Hochschulen durch Studiengebühren ${ }^{41}$ ), Lenkungszwecken, bei denen der Sach-

32 Vgl. zum Gebührenrecht BVerfGE 108, 1 (18); BVerfG, NVwZ 2013, S. 638 ff.; zu den Sonderabgaben BVerfGE 113, 128 (149 f.); 122, 316 (334 f.); 123, 132 (142); Reimer/Waldhoff et al., (FN 30), S. 60. Der Gesetzgeber muss den Gebührenzweck zudem hinreichend klar erkennen lassen, BVerfGE, ebd.108, 1 (18); BVerfG, NVwZ 2013, S. 638 ff. BVerfGE 50, 217 (226), 93, 319 (344); 97, 332 (345); 108, 1 (17); 113, 128 (148); P. Kirchhof, in: HStR V (FN 2), § 119 Rn. 26; Waldhoff, in: HStR V (FN 2), § 116 Rn. 86 f.

34 BVerfGE 49, 223 (228); 82, 159 (178); 92, 91 (115); 113, 128 (148); P. Kirchhof, in: HStR V (FN 2), § 119 Rn. 62 f.; Waldhoff, in: HStR V (FN 2), § 116 Rn. 90.

35 Dazu FN 31.

36 St. Rspr., zuletzt BVerfGE 123, 321 (141).

37 Vgl. Gawel/Schmidt, (FN 9), S. 27.

38 BVerfGE 123, 321 (142).

39 Nicht unpassend hieß es insoweit früher, die Homogenität müsse in der Rechts- und Sozialordnung materiell vorgegeben sein; vgl. BVerfGE 82, 159 (180).

40 Dazu Waldhoff, StuW 2002, S. 285 ff.; Reimer/Waldhoff et al., (FN 30), S. 59 f.

41 BVerfG, Beschl. v. 8. Mai 2013 - 1 BvL 1/08 -, Tz. 32 ff. 
zweck in einer durch die Zahlungspflicht veranlassten Verhaltensänderung besteht (z.B. Studierende durch Studiengebühren für Langzeitstudierende zur Einhaltung der Regelstudienzeit zu veranlassen ${ }^{42}$ ), und Sozialzwecken, bei denen die Verteilung der Finanzierungslast im Vordergrund steht (z.B. eine differenzierte Ausgestaltung der Studiengebühren, die Härten und soziale Barrieren vermeidet $\left.{ }^{43}\right)$.

Die Unterscheidung von Finanzierungszwecken und Lenkungszwecken muss allerdings aus finanzwissenschaftlicher Sicht irritieren, vor allem, wenn sie zur Abgrenzung verschiedener Abgabenarten verwandt wird. Denn für die Finanzwissenschaften sind Finanzierungsformen und Verhaltensanreize nicht nur systematisch verbunden. In gewisser Weise besteht sogar der ganze Sinn der ökonomischen Analyse nicht-steuerlicher Abgaben darin, diese Zusammenhänge zu erforschen, um diejenigen Finanzierungsformen zu identifizieren, die Anreize für die effiziente Produktion und Nutzung eines Gutes setzen. Die Beitragsfinanzierung des öffentlich-rechtlichen Rundfunks ist zum Beispiel gegenüber echten Benutzungsgebühren eine effizientere Form der Finanzierung dieser konkreten Sachaufgaben, weil letztere die Nutzung des Angebots unnötig beschränkt. Der abweichende Sprachgebrauch ist aber nur ein terminologisches, und kein sachliches Problem. Man sollte es freilich aufklären, um den Blick auf die eigentlichen Parallelen zwischen den juristischen und den ökonomischen Konzepten frei zu machen. Diese liegen auf einer anderen Ebene, nämlich zwischen den verfassungsrechtlichen Rechtfertigungsgründen und den finanzwissenschaftlichen Güterkategorien, die insoweit nicht nur Grundlage einer Effizienzanalyse sind, sondern relevante Strukturen der ökonomischen Realität abbilden ${ }^{44}$.

aa) Positive Externalitäten als Grundlagen der Finanzierungszwecke. Sieht man auf die staatlichen Aufgaben, die unter dem Kriterium der Finanzierungszwecke als finanzierte Aufgaben in den Blick genommenen werden, erweisen sich diese als Güter, die entweder dem Abgabenschuldner individuell zugute kommen (tatsächlich bei der Gebühr, der Möglichkeit nach beim Beitrag) oder die zugunsten einer homogenen Gruppe von Abgabenschuldnern verwendet werden (Sonderabgabe). Soweit diese Nutzenbeziehungen nicht nur beabsichtigt oder vorgeschoben sind, sondern tatsächlich bestehen, sollte es sich bei der Aufgabe aus Sicht der finanzwissenschaftlichen Kollektivgüterlehre um die Produktion von öffentlichen Gütern oder Klubgütern handeln oder um die Bereitstellung von privaten Gütern, die der Abgabenschuldner selbst nutzt oder die für ihn mit positiven Externalitäten, meritorischen Nutzendimensionen oder Verteilungszwecken verbunden sind. In diesen Fällen kann man von einer Gegenleistung im positiven Sinn sprechen, weil dem Abgabenschuldner tatsächlich, potentiell oder

42 BVerfG (Kammer), VR 2006, S. $287 \mathrm{f}$.

43 BVerfG, Beschl. v. 8. Mai 2013 - 1 BvL 1/08 -, Tz. 41 ff.

44 In vergleichbarer Weise zieht das BVerfG seit der Glykol-Entscheidung die „Funktionsbedingungen des Wettbewerbs“ für die Auslegung der Berufsfreiheit heran (wenngleich nicht immer an der dogmatisch überzeugenden Stelle); vgl. BVerfGE 105252 (265); 110, 274 (288); 115, 205 (152); 116, 135 (152). Es überträgt den Gedanken in der Entscheidung zu den kommunalen Hebesätzen auf die insoweit negativen Funktionsbedingungen eines kommunalen Steuerwettbewerbs, BVerfGE 125, 141 (169). Zur Bedeutung der Wirklichkeitsanalyse generell für die Auslegung der Grundrechte G. Kirchhof, Grundrechte und Wirklichkeit, 2007. 
als Mitglied einer Gruppe ein Nutzen zufließt. Der öffentlich-rechtliche Rundfunk fällt zum Beispiel in diese Kategorie. Interessant sind hier die Absatzfonds-Entscheidungen des BVerfG $^{45}$. Hier mussten Unternehmen der Land- und Ernährungswirtschaft und der Holzwirtschaft über Sonderabgaben Fonds finanzieren, die mit den Mitteln Werbemaßnahmen zugunsten der jeweiligen Industrie bezahlten. Da die begünstigten Unternehmen diese nicht selbst unternommen haben, fragt sich aber, ob es tatsächlich einen Bedarf für diese Werbemaßnahmen gab. Das ist nicht ausgeschlossen, weil industrieweite Werbung für die einzelnen Unternehmen den Charakter eines öffentlichen Gutes mit entsprechenden Kooperationsproblemen haben kann. Die Unternehmen könnten eine Kooperation dann aber immer noch über ihre Industrieverbände organisieren. Deshalb verlangt das BVerfG in solchen Fällen zu Recht, der Gruppennutzen müsse evident sein und die Betroffenen müssten das Problem voraussichtlich nicht selbst lösen können ${ }^{46}$. Der Gedanke sollte auf alle gruppenbezogenen öffentlichen Güter übertragen werden.

bb) Negative Externalitäten als Grundlage der Lenkungszwecke. Verhaltenslenkung ist, wie gesagt, aus ökonomischer Sicht kein Spezifikum der sog. Lenkungsabgaben. Sieht man auf die Fälle, die unter dem Kriterium der (oft umweltrechtlichen) Lenkungszwecke angesprochen sind, handelt es sich in der Regel um Konstellationen, in denen die Verhaltenslenkung aus einem speziellen Anlass geschieht, der wiederum mit der ökonomischen Gutscharakteristik zusammenhängt. Der Zusammenhang ist hier aber nicht positiver, sondern negativer Art, nämlich dass der Abgabenschuldner (oder eine insoweit homogene Gruppe von Abgabenschuldnern) ein dem Staat gehörendes oder der Allgemeinheit zuzuordnendes Gut nutzt und dabei Ressourcen verbraucht ${ }^{47}$. Anlass der Abgabenerhebung ist mithin, dass der Abgabenschuldner die Nutzungsmöglichkeiten anderer Bürger mindert. Aus Sicht der finanzwissenschaftlichen Kollektivgutslehre handelt es sich deshalb in der Regel um Gemeingüter oder negative Externalitäten ${ }^{48}$. So liegt es bei der entgeltlichen Vergabe oder Versteigerung von Funklizenzen, die wie gesagt Externalitäten bei der Nutzung des Funkspektrums als eines Gemeinguts regulieren, aber auch bei Umweltabgaben, die auf den Gebrauch von natürlichen Ressourcen erhoben werden. In diesen Fällen geht es nicht um den Austausch von Leistungen zwischen Staat und Bürgern, sondern um die Einschränkung wechselseitiger Beeinträchtigungen, also nicht um Gegenseitigkeit im positiven Sinn, sondern vergleichbar dem Verursacherprinzip um Verantwortung für Beeinträchtigungen.

Insoweit der Nutzer bzw. Verursacher in diesen Fällen aber aus seinem für andere negativen Tun selbst einen positiven Nutzen zieht, besteht im weiteren Sinn ein Gegenseitigkeitsverhältnis, wie es sich auch im Begriff der Vorteilsabschöpfungsabgabe andeutet ${ }^{49}$. Reicht dies

45 BVerfGE 122, 316 (332 ff.); 123, 132 (139 ff.); dazu Germelmann, GewerbeArchiv 2009, S. 476 ff.; Rodi, JZ 2009, S. 689 f.

46 BVerfGE 122, 316 (338); 123, 132 (142 f.).

47 Zur Diskussion um die Umweltabgaben oben, FN 26.

48 Wobei negative Externalitäten auch darin bestehen können, dass der Bürger durch Nutzung eines vom Staat kostenfrei bereit gestellten privaten Gutes (z.B. der Hochschulen) die Nutzungsmöglichkeiten anderer Nutzer beeinträchtigt. 
zur Rechtfertigung, wird die Kategorie der Lenkungsabgabe freilich weitgehend obsolet ${ }^{50}$. Bei natürlichen Ressourcen trägt die Kosten der Ressourcennutzung allerdings die Allgemeinheit, während der Staat über die Verwaltungskosten hinaus keinen Kostenaufwand hat. Lehnt man aus diesem Grund eine Rechtfertigung über den Gegenleistungsgedanken ab, bleibt der Lenkungsgedanke erheblich ${ }^{51}$. Für eine im Sinne des allgemeinen Äquivalenzgedankens materiell angemessene Begrenzung der Abgabenerhebung sollte man aber in Betracht ziehen, den Gesichtspunkt der Vorteilsabschöpfung kumulativ zur Begrenzung von Lenkungszwecken anzuwenden, also Lenkungszwecke im Rahmen von nicht-steuerlichen Abgaben nur zuzulassen, wenn sie zugleich der Vorteilsabschöpfung dienen ${ }^{52}$, und den Gesetzgeber für darüber hinausgehende Lenkungszwecke auf Steuern zu verweisen.

cc) Ausgestaltende Sozialzwecke. Sozialen Zwecken als primärer Rechtfertigung nichtsteuerlicher Abgaben begegnet man vor allem im Sozialrecht und verwandten Materien, zum Beispiel in Form der Insolvenzsicherungsabgabe ${ }^{53}$. Wenn in anderen Bereichen unter dem Stichwort der „Ausgleichsabgaben“ Kosten auf Unternehmen umgelegt werden, geht es der Sache nach im günstigen Fall um eine Verantwortlichkeit für die Verursachung von Externalitäten, möglicherweise um Verhaltenslenkung ohne Vorteilsabschöpfung, die wie oben ausgeführt hier kritisch gesehen wird, mitunter aber auch nur um die Überwälzung der Finanzierung von „Gütern“, die der Allgemeinheit zugute kommen, auf Nicht-Verantwortliche, letztlich also um unzulässige Fiskalzwecke ${ }^{54}$. Häufig bilden soziale Zwecke aber nur ergänzende Zwecke im Rahmen der Ausgestaltung von Abgaben, die primär zur Finanzierung von „Gegenleistungen“ (im engeren oder weiteren Sinn) dienen (z.B. die soziale Ausgestaltung von Studiengebühren) ${ }^{55}$ oder Vorteile aus negativen Externalitäten abschöpfen (z.B. gestaffelte Wassergebühren $)^{56}$. Das sind legitime Verteilungsfragen, die methodisch als solche nicht Gegenstand der Wohlfahrtsökonomie sind. Was die ökonomische Analyse hier beitragen kann, ist mit den Fragestellungen vergleichbar, die die Finanzwissenschaften bei der Analyse von Steuern verfolgen, nämlich die tatsächlichen Verteilungswirkungen aufzuzeigen (Inzidenz) und die Wohlfahrtsfolgen der Verteilungsmechanismen zu analysieren. Flächendeckend gleiche Abfallgebühren führen zum Beispiel dazu, dass einkommensschwache Bewohner von Wohnhochhäusern (niedrige Sammlungskosten wegen hoher räumlicher Konzentration) die Abfallsammlung bei einkommensstarken Bewohnern von Villengebieten (hohe Sammlungskosten wegen geringer räumlicher Konzentration) quersubventionieren.

50 Das BVerfG konnte die Frage der Rechtfertigung aus der Lenkungsfunktion in der WasserpfennigEntscheidung entsprechend offenlassen, vgl. BVerfGE 93, 319 (345).

51 P. Kirchhof, in: HStR V (FN 2), § 119 Rn. 9 ff.

52 Dieser Gedanke klingt auch an in BVerfG (Kammer), VR 2006, 287 (288).

53 BVerfG, NZA 2013, S. 193 ff.

54 Instruktiv Reimer/Waldhoff et al., (FN 30), S. 49 ff.

55 Vgl. BVerfG, Beschl. v. 8. Mai 2013 - 1 BvL 1/08 -, Tz. 48, mit dem Hinweis, die Studiengebühren seien in Bezug auf die Gesamtkosten des Studiums geringfügig. Bezogen auf das gebührenrechtliche Gegenseitigkeitsverhältnis ,,übererfüllt“ der Staat also. Dafür lassen sich neben den Verteilungsgründen (Sozialstaat und Teilhabe), die das BVerfG in den Vordergrund stellt, aus ökonomischer Sicht auch noch positive externe Effekte und meritorische Zwecke ins Feld führen; vgl. Blankart, (FN 11), S. 80 f. 


\section{Private und kollektive Güter}

Grundlage für die weiteren Überlegungen ist die Kollektivgütertheorie, die kurz skizziert wird $^{57}$. Bekanntermaßen sollten private Güter aus wohlfahrtsökonomischer Sicht privat produziert und über Tauschgeschäfte auf Märkten auf die Konsumenten verteilt werden. Bei Freiwilligkeit der Produktions- und Konsumentscheidungen werden dadurch Angebot und Nachfrage über den Preismechanismus dezentral koordiniert, so dass die verbrauchten Ressourcen der Tendenz nach effizient genutzt werden. Anders als im Recht bezieht sich der Begriff "private Güter" hier aber nicht auf Güter, die im Eigentum Privater stehen, sondern auf Güter, die unabhängig von der Eigentumsordnung zwei ökonomisch relevante Eigenschaften aufweisen. Beide beziehen sich auf den Gebrauch eines Gutes, nämlich Ausschließbarkeit auf die Möglichkeit des Berechtigten, Unberechtigte vom Gebrauch auszuschließen, und Rivalität auf die Beeinträchtigung der weiteren Gebrauchsmöglichkeiten durch den Gebrauch. Die Kombination beider Eigenschaften bildet die Grundlage, dass private Güter auf Märkten tendenziell effizient bereitgestellt werden können ${ }^{58}$. Fehlt eine oder fehlen beide dieser Eigenschaften, kann man von einem Kollektivgut sprechen (die Terminologie ist insoweit nicht einheitlich). Fehlen Ausschließbarkeit und Rivalität, handelt es sich um ein (reines) öffentliches Gut. Ist Ausschließbarkeit gegeben, fehlt aber die Rivalität, spricht man von einem Klubgut (bzw. von einem Mautgut oder einem ausschließbaren öffentlichen Gut). Fehlt die Ausschließbarkeit, rivalisiert aber der Gebrauch des Gutes, handelt es sich um ein Gemeingut (bzw. um eine Almende).

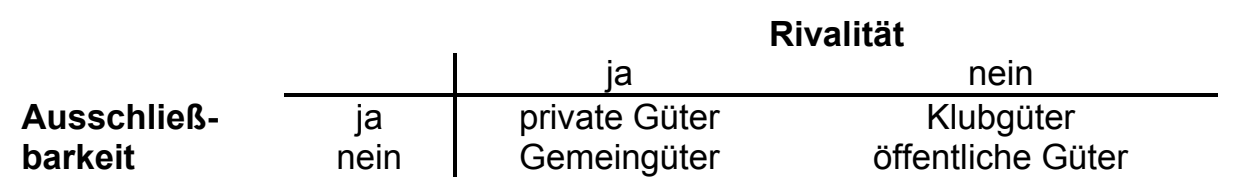

Tabelle: Private und kollektive Güter

Mit Blick auf die Rolle der nicht-steuerlichen Abgaben ist es sinnvoll, zwei Fragenkreise zu unterscheiden, nämlich einerseits Probleme hinsichtlich der Produktion kollektiver Güter und Probleme in Bezug auf die Nutzung des Gutes. Mangelnde Anreize für die private Produktion eines Kollektivguts entstehen nur im Fall der fehlenden Ausschließbarkeit, also bei öffentlichen Gütern und Gemeingütern. Dies wird zunächst erörtert (unten, III.). Für die Frage der effizienten Nutzung kommt es wesentlich auf die Rivalität bzw. Nicht-Rivalität des Gutes an. Von ihr hängt auch die Wahl der Ausgestaltung der Finanzierungsform ab. Fehlt die Rivalität im Gebrauch, wie bei öffentlichen Gütern, positiven Externalitäten oder Klubgütern, sollte eine Nutzung möglichst unbeschränkt zugelassen werden (unten, IV. 1.). Liegt Rivalität dage-

$57 \quad$ Nachweise s. FN 20.

58 Für die Zwecke dieses programmatischen Artikels werden die bekannten immanenten Probleme der Wohlfahrtstheorie und ihre Kritik von Seiten der Verhaltensökonomie zunächst zurückgestellt und pragmatisch unterstellt, dass sich auf ihrer Grundlage Tendenzaussagen über Anreize und Effizienzwirkungen formulieren lassen (vgl. Sutton, Marshall's Tendencies. What Can Economists Know?, 2002), die im Grundsatz von praktischer Relevanz sein können, aber im konkreten Anwendungsfall zu diskutieren und empirisch zu überprüfen sind. 
gen vor, wie bei Gemeingütern und negativen Externalitäten, sollte der Gebrauch auf ein ressourcenschonendes Maß beschränkt werden (unten, IV. 2.). Besondere Probleme stellen sich hinsichtlich der Nutzung von Mischgütern und privaten Gütern mit öffentlichen Zwecken (unten, V.).

\section{Zur staatlichen Produktion kollektiver Güter}

Ein Aspekt der Kollektivgütertheorie betrifft die Frage, wann privaten Unternehmen der Anreiz für die effiziente Produktion eines Gutes fehlt. Damit wird die Finanzierungsfunktion der nicht-steuerlichen Abgaben angesprochen, die aus ökonomischer Sicht allerdings unter der Fragestellung behandelt wird, ob eine staatliche Bereitstellung effizienter wäre ${ }^{59}$. NichtAusschließbarkeit und/oder Nicht-Rivalität können dafür Gründe sein.

\section{1. Öffentliche Güter}

a) Unzureichende Anreize für private Produktion. Ausschließbarkeit meint die faktische Möglichkeit, Dritte zu vertretbaren Kosten vom Konsum auszuschließen. Sie ist Voraussetzung für die Etablierung privater Verfügungsrechte ${ }^{60}$. Ohne Ausschlussmöglichkeit besteht für potentielle Konsumenten kein Anlass, für den Gebrauch des Gutes ein Entgelt zu errichten. Mangels Aussicht auf Entlohnung fehlt den potentiellen Produzenten dadurch der Anreiz zur Produktion des Gutes $^{61}$. Trotzdem kann die Produktion des Gutes aus ökonomischer Sicht aber wünschenswert sein, nämlich wenn der Nutzen, den die Konsumenten insgesamt aus dem Gut ziehen würden, die Produktionskosten übersteigt. Nicht-ausschließbare Güter werden deshalb auf Märkten nicht oder nicht in dem ökonomisch wünschenswerten Umfang bereitgestellt. Viele nicht-ausschließbare Güter, insbesondere solche mit einem größeren Nutzerkreis, werden dann vom Staat und seinen Untergliederungen bereitgestellt. Denn der Staat ist nicht darauf angewiesen, die Kosten der Produktion eines öffentlichen Gutes über freiwillig gezahlte Entgelte zu decken, sondern kann sie mit Hilfe seiner Zwangsbefugnisse eintreiben, insbesondere durch die Erhebung von Steuern oder nicht-steuerlichen Abgaben ${ }^{62}$.

b) Ausschlusstechnologien und gewollter Nicht-Ausschluss. Ob Dritte in ökonomisch relevanter Weise vom Gebrauch ausgeschlossen werden können, ist nicht unmittelbar eine Frage der physischen Eigenschaften des Gutes, sondern der verfügbaren Ausschlusstechnologien und ihrer Kosten ${ }^{63}$. Fernsehsendungen zum Beispiel wurden mit der Einführung bezahlbarer Verschlüsslungstechnologien von einem nicht-ausschließbaren öffentlichen Gut zu einem aus-

59 Die Anreize des Staates für eine wohlfahrtsoptimale Bereitstellung werden in diesem Rahmen allerdings kaum thematisiert; vgl. Hellwig, (FN 20), S. 34 ff.

60 Nowotny/Zagler, (FN 20), S. $60 \mathrm{ff}$.

61 Dieses Produktionsproblem wird vor allem für die reinen öffentlichen Güter diskutiert, kann aber im Prinzip auch bei Gemeingütern auftreten, solange es sich nicht um natürliche Ressourcen handelt.

62 Mitunter sind dann zivil-gesellschaftliche, also private, aber nicht-marktliche Lösungen möglich, die oft auf informalem Zwang oder nicht-eigennützigen Motiven beruhen, etwa wenn die Mitglieder einer Kirchengemeinde eine Orgel über Spenden finanzieren. 
schließbaren Klubgut. Dass bedeutet aber nicht, dass der Staat, wenn er Güter bereitstellt, von einer Ausschlussmöglichkeit immer Gebrauch macht. Der öffentlich-rechtliche Rundfunk, aber etwa auch der Gemeingebrauch an öffentlichen Straßen, sind Beispiele, in denen der Staat eine entgeltfreie öffentliche Nutzung des Gutes gestattet und die Produktion über Zwangsabgaben (Rundfunkbeiträge bzw. Steuern) finanziert ${ }^{64}$. Auch dafür kann es ökonomische Gründe geben, die aber nicht mit Problemen bei der Finanzierung des Gutes zusammenhängen, sondern mit der Ineffizienz, weitere Nutzer vom Gebrauch auszuschließen, wenn der Gebrauch nicht rivalisiert (dazu im nächsten Abschnitt). Ein wesentlicher Unterschied bei einer öffentlichen Bereitstellung von Gütern, bei denen ein Ausschluss technisch und kostenmäßig möglich ist, besteht allerdings darin, dass dann private Konkurrenz möglich ist (und beim Rundfunk z.B. in Gestalt des Bezahlfernsehens auch existiert). Die ökonomische Analyse wird dann durch die Interaktion zwischen privater und öffentlicher Bereitstellung verkompliziert.

c) Natürliche Ressourcen. Die Frage der Produktion und ihrer Finanzierung stellt sich natürlich nur, soweit ein Kollektivgut überhaupt produziert werden muss. Daran fehlt es zum Beispiel bei der im juristischen Schrifttum gerne zitierten Luft, die wie andere natürliche Ressourcen - Grundwasser oder Fischbestände - jedenfalls in bestimmtem Umfang bereits vorhanden ist. Solche Ressourcen werfen, auch wenn insoweit die Ausschließbarkeit fehlt, kein Produktionsproblem auf ${ }^{65}$. Sie kämpfen aber möglicherweise mit Übernutzungsproblemen, nämlich wenn es sich um Gemeingüter handelt, deren Nutzung rivalisiert.

\section{Private Güter mit positiven Externalitäten, meritorischen Nutzendimen- sionen und Verteilungszwecken}

Vor allem in den unter dem Stichwort der Daseinsvorsorge geführten Bereichen, aber auch anderswo, stellt der Staat den Bürgern eine Vielzahl von Gütern bereit, die im Hinblick auf ihre unmittelbare Nutzung eigentlich private, ausschließbare und rivalisierende Güter sind, aber aus Gründen des öffentlichen Interesses nicht über Märkte, sondern vom Staat und insbesondere den Kommunen bereitgestellt werden. Darunter fallen keineswegs nur nichtwirtschaftliche Betriebe wie Theater oder Museen, sondern auch der große Bereich der wirtschaftlichen Betätigung der öffentlichen Hand. Soweit dahinter nicht (verdeckte) rein erwerbswirtschaftliche Zwecke stehen, kann man die öffentlichen Interessen an einer solchen staatlichen Bereitstellung privater Güter aus ökonomischer Sicht vor allem unter drei Aspekten abbilden: positive Externalitäten, meritorische Nutzendimensionen und Verteilungszwecke.

a) Positive Externalitäten. Ökonomisch betrachtet sind nicht-ausschließbare Güter ein Sonderfall positiver Externalitäten. Sie stiften einen Nutzen, der nicht Gegenstand einer Markt-

64 Zur Ausschließbarkeit bei öffentlichen Straßen Gawel/Schmidt, (FN 9), S. 27; Benson, Are Roads Public Goods, Club Goods, Private Goods, or Common Pools?, Working Paper, Florida State University. 
transaktion ist. Solche positive Externalitäten treten aber nicht nur als öffentliche Güter, sondern auch als zusätzliche Nutzendimension privater Güter auf, die als solche über den Markt bereitgestellt werden oder bereitgestellt werden könnten ${ }^{66}$. Hochschulen bieten, soweit sich die Hochschulbildung in einem höheren Einkommen niederschlägt, ein privates Gut an ${ }^{67}$. Man erhofft sich von ihrer Inanspruchnahme aber auch positive Wirkungen durch geringere Kriminalität, niedrigere Gesundheitskosten oder eine Akkumulation von Humankapital ${ }^{68}$. Auch hinsichtlich dieser öffentlichen Nutzungsdimensionen privater Güter gilt, dass sie vom Markt in zu geringem Umfang produziert werden, weil der private Kunde nicht bereit ist, für den Nutzen der Allgemeinheit zu zahlen.

b) Meritorische Nutzendimensionen. Mit diesem Problem kämpfen definitionsgemäß auch sog. meritorische Güter wie Schulen, Theater, Museen oder der öffentlich-rechtliche Rundfunk, bei denen die private Nachfrage aus Sicht des Gemeinwohls zu gering ist ${ }^{69}$. Auch ihnen liegen häufig private oder zumindest ausschließbare Güter zugrunde, und auch ihnen eignet eine zusätzliche Dimension, für die die Nutzer keine (ausreichende) Zahlungsbereitschaft zeigen, so dass die Produktionskosten insoweit anders als über Entgelte finanziert werden müssen. Worin diese meritorische Dimension besteht, ist allerdings unklar und wird recht heterogen gesehen ${ }^{70}$. Sinnvollerweise werden über sie vor allem paternalistische Interventionen aufgrund nicht-rationalen Verhaltens oder nicht-rationaler, insbesondere zeit-inkonsistenter Präferenzen angesprochen ${ }^{71}$, zum Beispiel dass potentielle Studenten die in der Zukunft liegenden Vorteile durch ein höheres Einkommen irrational unterschätzen. Aber auch unabhängig davon kann der Staat aus Gemeinwohlgründen, die nicht an die Präferenzen der Bürger anknüpfen, bestimmte Nutzungen für gewünscht erklären, wie er es etwa beim öffentlichen Rundfunk im Hinblick auf die für die Demokratie essentielle politische Meinungsbildung tut, für die Bürger nach einem Hochschulstudium möglicherweise besser informiert sind. Für das hiesige Thema der Finanzierung durch nicht-steuerliche Abgaben ist insoweit entscheidend, dass, wenn der Staat in rechtlich zulässiger Weise beschließt, ein Gut solle auch in seinen meritorischen Dimensionen bereitgestellt werden, er nicht davon ausgehen kann, dass diese Bereitstellung von privaten Märkten in ausreichendem Maß gewährleistet wird. Die Gründe fehlende Zahlungsbereitschaft der Nutzer - sind strukturell die gleichen, wie bei positiven Externalitäten.

c) Verteilungszwecke. Wie bei meritorischen Gründen zielt die durch Verteilungszwecke motivierte staatliche Bereitstellung von privaten Gütern regelmäßig darauf ab, die Nachfrage nach einem Gut zu erhöhen, das aus öffentlicher Sicht auf Märkten zu gering nachgefragt

66 Musgrave/Musgrave, (FN 11), S. 49.

67 Blankart, (FN 11), S. 80.

68 Blankart, (FN 11), S. 80; Musgrave/Musgrave (FN 11), S. 183.

69 Musgrave/Musgrave, (FN 11), S. 55 ff. (,merit goods“).

70 Positive externe Effekte sollten als solche thematisiert werden. Auch fehlende oder asymmetrisch verteilte Informationen werfen spezifische Fragen auf, die sinnvollerweise gesondert analysiert werden.

71 Zimmermann/Henke/Broer, (FN 11), S. 55 ff.; zur Verhaltensökonomik Thaler/Sunstein, Nudge, 2008; Englerth, in: Towfigh/Petersen (Hrsg.), Ökonomische Methoden im Recht, 2010, S. 165 ff.; zum Paternalismusproblem van Aaken, in: Anderheiden et al. (Hrsg.), Paternalismus und Recht, 2006, S. 109 ff.; Englerth, in: Engel et al. (Hrsg.), Recht und Verhalten, 2007, S. 231 ff. 
wird, weil die Zahlungsbereitschaft zu gering ist. Der Grund für die mangelnde Zahlungsbereitschaft liegt aber nicht in der individuellen Wertschätzung für den Gebrauch des Gutes, sondern in dem unzureichenden Einkommen mancher Nutzer. Universaldienstleistungen und soziale Gebührenstafflungen sind Beispiele dafür. In beiden Fällen werden zwar entgeltähnliche Abgaben erhoben, die Gebührenhöhe liegt aber (für einen Teil der Nutzer) unter den Produktionskosten. Wiederum liegt das Problem in der Unterproduktion, jetzt weil die Entgelte aus Verteilungsgründen zu gering sind.

d) Staatliche Produktion oder Subvention. Wie bei öffentlichen Gütern kommt bei der Verknüpfung privater Güter mit öffentlichen Externalitäten, meritorischen Nutzendimensionen oder Verteilungszwecken eine staatliche Intervention in Betracht. Was die Bereitstellung dieser Güter anbetrifft, besteht aber im Vergleich zu öffentlichen Gütern ein für die Praxis ganz wesentlicher Unterschied, der auch im Recht an verschiedenen Stellen für erhebliche Friktionen sorgt. Während bei echten öffentlichen Gütern eine marktförmige Bereitstellung praktisch nicht möglich ist, stehen dem Staat bei privaten Gütern mit positiven Externalitäten mehrere Möglichkeiten offen. Er kann die Produktion in privater Hand lassen und versuchen, das Problem einer Unterproduktion durch Subventionen an die privaten Produzenten auszugleichen. Oder er kann die Produktion in staatliche Hand nehmen, sollte dann aber etwa über Entgelte oder Gebühren die privaten Nutzenvorteile abschöpfen.

\section{Probleme einer staatlichen Produktion}

Die staatliche Bereitstellung öffentliche Güter (und private Güter mit öffentlichen Zwecken) ist freilich selbst nicht frei von Problemen. Dafür gibt es strukturelle Gründe, darunter nicht zuletzt, dass bei kollektiven Gütern der Markt als Mechanismus zur verschwendungsarmen Nutzung von Ressourcen eben nicht zur Verfügung steht. Der ökonomischen Analyse fällt insoweit die Aufgabe zu, Möglichkeiten aufzuzeigen, um unnötiges Staatsversagen zu vermeiden. Dieses Anliegen prägt aber im Grund alle Felder des modernen Wirtschaftsverwaltungsrechts - Privatisierung, Regulierung, Vergabe, Beihilfen, usw. -, wobei nicht-steuerliche Abgaben nur punktuell eine Rolle spielen.

a) Nicht-steuerliche Abgaben und Äquivalenz. Ein Problem einer abgabenfinanzierten Bereitstellung kollektiver Güter ist die Gefahr, dass der Staat an den Bedürfnissen der Bürger vorbei produziert. So kann er bei einer Finanzierung über nicht-steuerliche Abgaben die Entdeckungsfunktion von Märkten ${ }^{72}$ nicht nutzen, um die Präferenzen der Bürger zu ermitteln. Versuche, diese Informationen auf andere Weise zu erhalten, sind nicht ohne methodische und normative Schwierigkeiten ${ }^{73}$. Dieses Problem wird gemildert, wenn ein Gut nach dem Äqui-

72 von Weizsäcker, in: ORDO 56 (2005), S. 95 ff. Zwar sind auch auf Märkten mit privaten Gütern die Informationen über die Zahlungsbereitschaft zum Teil asymmetrisch verteilt. Aber Konkurrenz zwischen den Nachfragern macht es für diese schwieriger, die Unkenntnis der Produzenten strategisch zur Manipulation der Preise auszunutzen, vgl. Hellwig, (FN 20), S. 20.

73 Bei Wahlen und Abstimmungen bestehen Anreize, seine Präferenzen falsch zu offenbaren, und die Praktikabilität der zur Bewältigung dieses Problems ersonnenen spieltheoretischen Mechanismen ist fraglich; vgl. Cornes/Sandler, (FN 19), S. 198 ff.; Hindriks/Myles, (FN 20), S. 163 ff.; Mueller, (FN 20), S. 159 ff.; 
valenzprinzip finanziert wird und dessen Inanspruchnahme freiwillig ist, also kein Benutzungszwang besteht. In diesem Fall müssen die Abgaben nach der empfangenen Gegenleistung bemessen werden ${ }^{74}$. Den Wert der Gegenleistung kann man wohlfahrtsökonomisch fassen als empfangenen Nutzen ${ }^{75}$ oder abgabenrechtlich als Kostenaufwand ${ }^{76}$. Wegen der Freiwilligkeit kommt es in beiden Fällen nicht $\mathrm{zu}$ einer Beanspruchung über der Zahlungsbereitschaft, etwa wenn man den kommunalen Kindergarten einer teureren gewerblichen Alternative vorzieht. Bei freiwilliger Nutzung gilt dies im Grundsatz selbst dann, wenn es an Konkurrenzangeboten privater Produzenten mangelt, wie es bei öffentlichen Gütern oder einer durch Monopolrechte oder faktische Monopole geschützten staatlichen Bereitstellung privater Güter mit öffentlichen Zwecken der Fall ist. Bei nicht-rivalisierenden Gütern (wie dem Rundfunk) ist eine Gebührenfinanzierung bei freiwilliger Inanspruchnahme allerdings mit Blick auf eine möglichst effiziente Nutzung des Gutes problematisch, weil sie die Bürger in unnötiger Weise von dem Gebrauch des Gutes abhält. Dann kann eine Finanzierung über Beiträge, Pauschalabgaben oder Steuern vorzugswürdig sein.

Äquivalenz im finanzwissenschaftlichen Verständnis lässt dabei durchaus zu, einzelne Nutzer auch über ihrem anteiligen Kostenanteil zu belasten, solange die Abgaben ihre Zahlungsbereitschaft für das Gut nicht überschreiten. Eine Gebührenstaffelung im Sinne einer „Preisdiskriminierung" nach Zahlungsbereitschaft kann insbesondere in Betracht kommen, wenn die Nachfrage nach dem Gut vom Einkommen abhängt ${ }^{77}$. Die Gebührenerhebung erfährt dann eine Differenzierung nach Leistungsfähigkeit ${ }^{78}$, wie bei der sozialen Staffelung von Kindergartenbeiträgen $^{79}$. Im Hinblick auf die unterschiedliche Wertschätzung widerspricht dabei auch eine progressive Staffelung mit einer Kostenbelastung über den anteiligen Kosten der finanzwissenschaftlichen Äquivalenz nicht. Auch das BVerfG hält insoweit eine Kostenüberdeckung mit Blick auf den „Wert der Leistung für den Empfänger“ für möglich ${ }^{80}$.

b) Fehlender Wettbewerb. Bei der Bereitstellung kollektiver Güter ist der Staat oft keinem Wettbewerb ausgesetzt. In Monopolbereichen fehlt es bereits an Wettbewerbern. Wenn das Gut nicht ausschließlich über Benutzungsgebühren finanziert wird, sondern auch aus Steuermitteln oder über Beiträge oder Sonderabgaben, ist der Staat privatem Konkurrenzdruck weniger ausgesetzt. Da der Staat aber nicht als gewinnmaximierendes, auf größtmögliche Monopolrente abzielendes Unternehmen agiert (agieren darf), sondern im öffentlichen Interesse tätig wird (werden sollte), sind staatliche Monopole in dieser Hinsicht gegenüber privaten

Siehe aber die Arbeiten von Martin Hellwig und Kollegen, vgl. Hellwig, (FN 20). Daneben gibt es empirische Methoden, die das Informationsproblem zumindest mildern Pommerehne, Präferenzen für öffentliche Güter, 1987; Revesz/Stavins, in: Polinksy/Shavell (Hrsg.), Handbook of Law and Economics, S. 499 (508 ff.); Schläpfer/Zweifel, Wirtschaftsdienst 88 (2008), S. 210 ff.

76 Brüning, in: Driehaus (Hrsg.), Kommunalabgabenrecht, 46. Erg. Lfg. 2012, § 6 Rn. 49b; Kaufmann, in: Henneke/Pünder/Waldhoff (Hrsg.), Recht der Kommunalfinanzen, 2006, § 15 Rn. 51 ff.

77 Vgl. Blankart, (FN 11), S. 190 ff. (für nicht-rivalisierende Güter).

78 Kritisch dazu Gawel, (FN 22), S. $194 \mathrm{f}$.

79 Zur rechtlichen Zulässigkeit siehe BVerfGE 97, 332 (344 ff.); BVerfG, NVwZ 1995, S. 173 ff., NVwZ 1995, S. 790 ff., NJW 2000, S. 1129 ff.; Jeastaedt, in: DVBl. 2000, S. 1820 ff. 
Monopolisten möglicherweise weniger problematisch ${ }^{81}$. Der Staat unterliegt aber wohl noch stärker als ein privater Monopolist der Tendenz, zu überhöhten Kosten zu produzieren (sog. $\mathrm{X}$-Ineffizienzen) ${ }^{82}$. Die Frage, wie der Staat trotz mangelnder Gewinnorientierung kosteneffizient arbeiten kann, ist freilich ein eigener, weiter Fragenkreis ${ }^{83}$. Er berührt aber zum Beispiel das hiesige Thema, wenn der Staat private Güter öffentlich-rechtlich und über Gebühren finanziert bereitstellt, wie es bei der Trinkwasserversorgung häufig vorkommt ${ }^{84}$. Dann stellt sich die Frage, ob eine stärkere Kostenkontrolle der Gebühren des staatlichen Versorgers wünschenswert ist. In Betracht kommt dafür eine abgabenrechtliche Kontrolle nach Maßgabe von Äquivalenz und Erforderlichkeit ${ }^{85}$, die aufgrund des neuen $\S 130$ Abs. 1 S. 2 GWB nach der gegenwärtigen Rechtslage alleine noch möglich ist, eine kartellrechtliche Missbrauchskontrolle, die vom Bundeskartellamt gefordert wurde ${ }^{86}$ und deren rechtliche Zulässigkeit bis zur 8. GWB-Novelle umstritten war $^{87}$, oder eine Regulierung der Preise, die in einigen europäischen Ländern für die Wasserwirtschaft praktiziert wird ${ }^{88}$ und welche die Monopolkommission, wofür viel spricht, wegen der beim Wassertransport bestehenden natürlichen Monopole auch für Deutschland vorgeschlagen hat ${ }^{89}$. Das (jetzt nur noch rechtspolitische) ökonomische Sachproblem ist, ob eine kartell- oder regulierungsrechtliche Bekämpfung monopolbedingter Ineffizienzen die Qualität der Wasserversorgung beeinträchtigen oder andere unerwünschte Nebenfolgen haben würde. Insoweit käme es vor allem darauf an, worin genau die zu beobachtenden hohen Differenzen der Wassergebühren und insbesondere der in sie eingestellten unspezifischen Gemeinkosten ihre Ursache haben und welche Wirkungen eine Kartellaufsicht oder verschiedene Regulierungsformen haben würden ${ }^{90}$. De lege lata sollte sich der Blick aber vor allem auf die Verbesserung der abgabenrechtlichen Kostenkontrolle richten, deren Wirksamkeit auch von den richterrechtlich bestimmten Kontrollmaßstäben und der Aufsichtspraxis und ihren institutionellen Rahmenbedingungen abhängt ${ }^{91}$. Sinnvoll erscheint insbesondere eine am Wirtschaftlichkeitsgebot ausgerichtete Auslegung der Erforderlichkeitsprüfung, die auch manifeste Produktionsineffizienzen in den Blick nimmt ${ }^{92}$. Welche

$81 \quad$ Nowotny/Zagler, (FN 20), S. 97.

82 Hellwig, (FN 20), S. 35; für private Unternehmen Motta, Competition Policy, 12. Aufl. 2009, S. 45 f.; Schwalbe/Zimmer, Kartellrecht und Ökonomie, 2. Aufl. 2011, S. 20.

83 Ein Schritt ist zum Beispiel die Budgetierung des Neuen Steuerungsmodells vgl. Korioth, in: HoffmannRiem/Schmidt-Aßmann/Voßkuhle (Hrsg.), Grundlagen des Verwaltungsrechts III, 2009, § 44 Rn. 57 ff. Zur Behandlung Governance-Problemen im Rahmen der Kollektivguttheorie vgl. Hellwig, (FN 20), S. 34 ff.

84 Zum Ordnungsrahmen Brehme, Die Privatisierung und Regulierung der öffentlichen Wasserversorgung, 2010, zur Marktstruktur ebd. S. 62 ff.

85 Schulte/Wiesemann und Brüning, in: Driehaus (Hrsg.), Kommunalabgabenrecht, 30. Erg.Lfg 2004 bzw. 43. Erg.Lfg 2010, Rn. 69 ff.

86 Bundeskartellamt, Stellungnahme zum Referentenentwurf zur 8. GWB-Novelle, S. 19 ff.

87 Zur Diskussion bis zur Novelle vgl. Breuer, NVwZ 2009, S. 1249 ff.; Brüning, Zeitschrift für Wasserrecht 51 (2012), S. 1 ff.; Säcker, NJW 2012, S. 1105 (1108 ff.); Wolf, WuW 2003, S. 246 ff.; Wolfers/Wollenschläger, WuW 2013, S. 237 ff.; offen gelassen von BGH, NJW 2012, S. 1150 ff.

88 Meran, (FN 56), S. 167 ff.

89 Monopolkommission, Hauptgutachten 2008/2009, BT-Drucks 17/2600, S. 19 und 49 ff.; dies., Sondergutachten 63, S. $43 \mathrm{ff}$.

90 Dazu Meran, (FN 56).

91 Auf letzteres weisen hin Säcker, (FN 87), S. 1109; Monopolkommission, Sondergutachten (FN 89), S. 44.

92 Vgl. Brüning, (FN 87), S. 13; Schulte/Wiesemann und ders., (FN 85), Rn. 71. 
Aussichten eine solche Kostenkontrolle hätte und wie sie in die Kostenkonzepte des Abgabenrechts einzupassen wäre, wäre ein Aufgabe für eine eingehende ökonomische Analyse.

c) Vorhandener Wettbewerb. Dass fehlender Wettbewerb Probleme aufwirft, bedeutet andererseits nicht, dass Wettbewerb, wo er vorhanden ist, immer vorteilhaft wäre. Interferenzen ergeben sich insbesondere, wenn das Angebot an privaten Gütern, die im Blick auf öffentliche Zwecke subventioniert oder staatlich bereitgestellt sind, in Konkurrenz zu privaten Gütern tritt, die ohne die öffentliche Zielrichtung auf dem Markt angeboten werden. So konkurrieren öffentlich-rechtlicher Rundfunk und private Rundfunkanbieter oder kommunale Schwimmbäder mit privaten Spaßbädern. In diesen Fällen kann die staatliche oder staatlich subventionierte Bereitstellung die Wettbewerbsposition (anderer) privater Wettbewerber beeinträchtigen, etwa wenn der öffentlich-rechtliche Rundfunk Unterhaltungssendungen gebührenfinanziert ohne Werbepausen ausstrahlt. Umgekehrt kann aber auch die private Konkurrenz die Refinanzierungsmöglichkeiten ${ }^{93}$ oder die Nutzung der öffentlichen Bereitstellung stören, etwa wenn die Unterhaltungssendungen im privaten Fernsehen den anspruchsvollen politischen Sendungen im öffentlich-rechtlichen Rundfunk vorgezogen werden.

\section{Sonderkonstellationen der Bekämpfung öffentlicher Übel}

Ein klassisches Beispiel für ein nicht-ausschließbares Kollektivgut ist die von der Polizei geschützte öffentliche Sicherheit. Dieses Gut weist freilich, wie viele Regelungszwecke des allgemeinen Ordnungsrechts und des Sonderordnungsrechts, eine Besonderheit auf. „Gefahrenabwehrgüter" werden nämlich zu einem guten Teil durch Eingriffsrecht bereitgestellt, also dadurch, dass die Freiheitsausübung anderer Bürger beschränkt wird. Polizei und Straßenverkehrsbehörden sorgen etwa für die Sicherheit und Leichtigkeit des Straßenverkehrs im Wesentlichen dadurch, dass sie die den Straßenverkehrsteilnehmern obliegenden gesetzlichen Beschränkungen durchsetzen. Produktionskosten für ein öffentliches Gut entstehen hier nur insoweit die Gefahrenabwehr Verwaltungskosten verursacht, etwa für die Besoldung der befassten Amtsträger oder zum Erwerb der erforderlichen Ausrüstung. Der eigentliche Nutzen des Gutes, die „Sicherheit“, besteht aber in einem verhinderten Schaden, indem die von anderen Bürgern verursachten (bzw. von Bürgern wechselseitig ausgeübten) negativen Externalitäten begrenzt wurden. Das aber ist ein Gemeingutsproblem bzw. ein Problem negativer Externalitäten.

93 Zu den diesbezüglichen Problemen aufgrund der abgabenrechtlichen Restriktionen bei der Gebührengestaltung Gawel, (FN 1). 


\section{Nicht-steuerliche Abgaben und die Nutzung kollektiver Güter}

Für die Wahl und Ausgestaltung der Finanzierungsform ist der zweite Aspekt der Kollektivgütertheorie bedeutsam. Ziel ist hier eine effiziente Nutzung des Gutes. Angesprochen wird damit die Verknüpfung von Finanzierungsfunktion und Lenkungsfunktion. Die entscheidende Variable ist dabei Rivalität oder Nicht-Rivalität.

\section{Nutzungsanreize und Verteilungswirkungen bei Rivalität (Gemeingüter und negative Externalitäten)}

a) Gebrauch und Ressourcenverzehr. Der Konsum eines Gutes rivalisiert, wenn der Gebrauch einer Einheit eines Gutes durch eine Person den Gebrauch derselben Einheit des Gutes durch eine andere Person ausschließt oder beeinträchtigt. Das Brot, das eine Person gegessen hat, kann eine andere nicht mehr verzehren. Da der Konsum Ressourcen verbraucht, sollte er die Knappheit der für die Produktion des Gutes verbrauchten Ressourcen berücksichtigen, die in den Produktionskosten zum Ausdruck kommt. Bei privaten Gütern, die auf Märkten bereitgestellt werden, muss der Konsument die Produktionskosten bei seiner Konsumentscheidung berücksichtigen, weil er für das Recht zum Konsum einen Preis entrichten muss und darüber die Produktionskosten mitträgt. Eigentumsrechte sorgen auf diese Weise für die knappheitsgerechte Nutzung eines Gutes.

b) Rivalisierende Güter ohne Ausschluss (Gemeingüter). Bei Gemeingütern, deren Gebrauch rivalisiert, bei denen aber mangels Ausschließbarkeit keine privaten Eigentumsrechte möglich sind, wird die knappheitsgerechte Nutzung des Gutes zum Problem ${ }^{94}$. Ohne staatliche Intervention werden diese Güter tendenziell übernutzt, weil Nutzer den von ihnen verursachten Ressourcenverbrauch nicht internalisieren müssen. Gemeingüter bilden insoweit einen Sonderfall des allgemeinen Problems öffentlicher negativer Externalitäten, das unter dem Stichwort der Bekämpfung öffentlicher Übel bereits angesprochen wurde. Aus ökonomischer Sicht besteht hier eine zentrale Aufgabe des Staats darin, den Gebrauch von Gemeingütern (bzw. die Verursachung negativer Externalitäten im Allgemeinen) soweit zu beschränken, dass der durch ihn verursachte Ressourcenverbrauch berücksichtigt wird, also die sozialen Kosten der Nutzung zu internalisieren. Für diese Aufgaben kommen ordnungsrechtliche wie abgabenrechtliche Instrumente in Betracht. Das ist aus dem Umweltrecht wohl bekannt, wo als Alternative zu Umweltstandards auch Lenkungssteuern, Sonderabgaben mit Lenkungszwecken oder Ressourcennutzungsgebühren erhoben werden ${ }^{95}$.

c) Funktion und Wirkung von Abgaben bei Gemeingütern und negativen Externalitäten. Soweit der Staat nicht-steuerliche Abgaben zur Begrenzung sozialer Kosten erhebt, die aus der Nutzung von Gemeingütern oder durch negative Externalitäten entstehen, verfolgt die Kos-

94 Musgrave/Musgrave, (FN 11), S. $43 \mathrm{f}$

95 Statt Vieler Sacksofsky, (FN 26); dies., in: Hoffmann-Riem/Schmidt-Aßmann/Voßkuhle (Hrsg.), Grundlagen des Verwaltungsrechts, Bd. II 2008, § 40 Rn. 52 ff. 
tenbelastung den Lenkungszweck, die Übernutzung oder das öffentliches Übel einzudämmen. Die Belastung mit den sozialen Kosten dient dabei als Anreiz und damit als Mittel, um diesen Lenkungszweck zu erreichen. Ökonomisch wünschenswert wäre insoweit eine Ausgestaltung als Pigou-Steuer. Praktisch möglich sind aber nur Lenkungs- oder Demeritorisierungsabgaben, ${ }^{96}$ die in der Regel unterhalb einer Internalisierung der vollen sozialen Kosten bleiben. In diesen Umfang belasten sie den Abgabenschuldner zugleich und schöpfen insoweit auch die Vorteile ab, die dieser aus der Gemeingutsnutzung gezogen hat.

Diesen Vorteilen steht zwar kein staatlicher Aufwand gegenüber, wie er mit Verwaltungsgebühren gelten gemacht wird, ${ }^{97}$ also keine betriebswirtschaftlichen Kosten der befassten Behörde. Volkswirtschaftlich werden durch den Gemeingutsgebrauch bzw. die negativen Externalitäten aber reale Werte vernichtet. Im Sinne einer Entgeltung dieses durch die Nutzung von Gemeingütern verursachten Ressourcenverbrauchs besteht also durchaus ein Entsprechungsverhältnis. ${ }^{98}$ Es bleibt nur unklar, bei wem genau diese volkswirtschaftlichen Kosten anfallen, weil es sich bei den betroffenen Gemeingütern in der Praxis häufig um natürliche Ressourcen handelt (Luft, Flüsse, Grundwasser) und weil die von negativen Externalitäten betroffenen privaten Güter einer unklaren Vielzahl von Personen zugehören. Das Gegenleistungsverhältnis wird bei diesen Abgaben also gewissermaßen in ein Dreiecksverhältnis zwischen dem Bürger, dem Staat und der Allgemeinheit aufgespalten, in dem der Staat einen Ausgleich in Empfang nimmt, der für den bei der Allgemeinheit anfallenden Ressourcenverbrauch gezahlt wird.

d) Wettbewerbswirkungen und Kostenüberwälzung. Sind Ressourcennutzungsgebühren von Unternehmen zu tragen, steigen aufgrund der Vorteilsabschöpfung zugleich deren Produktionskosten, die dann je nach Marktstruktur über die Preise auf die Konsumenten übergewälzt werden und den Konsum verringern können. Volkswirtschaftlich kann das ein sinnvoller Effekt sein, weil der Konsum dann den tatsächlichen Ressourcenverbrauch internalisiert. Die Kostenbelastung kann aber für die Unternehmen zum Problem werden, wenn sie mit Unternehmen aus anderen Jurisdiktionen im Wettbewerb stehen, die keinen vergleichbaren Abgabepflichten unterliegen, und sie kann politisch zum Problem werden, wenn Preissteigerungen wesentliche Güter der Daseinsvorsorge betreffen.

96 Aus ökonomischer Sicht sollte die Bemessung der Abgabe möglichst die externen, sozialen Kosten internalisieren (Pigou-Steuer), was aber wegen des hohen Informationsbedarfs praktisch unmöglich ist. Nach dem Preis-Standard-Ansatz (Lenkungsabgabe) wird ein Verschmutzungsstandard politisch gesetzt und die Höhe der Gebühr an diesem Lenkungszweck ausgerichtet. Zum Teil werden Abgaben ohne operable Zieldefinition nur mit einer bestimmten Steuerungsrichtung erhoben (Demeritorisierungsabgaben). Übersichtlich dazu Gawel et al., (FN 26), S. 71 f.

97 Gebühren für Amtshandlungen oder sonstige Verwaltungstätigkeiten; vgl. zum Kommunalabgabenrecht Quaas, Kommunales Abgabenrecht, 1997, S. 18; zum Hochschulrecht BVerfGE 108, 1 (18 ff.), BVerfG, NVwZ 2013, S. 638 ff.

98 Insoweit geht das mitunter als argumentum ad absurdum angeführte hypothetische Beispiel einer Gebühr für Atemluft fehl. Denn auf der Erdoberfläche (aber nicht in Raumstationen) ist Luft in solchem Ausmaß vorhanden, dass deren Gebrauch als Atemluft praktisch nicht rivalisiert, Atemluft ist mithin kein Gemeingut, sondern ein reines öffentliches Gut, dessen Gebrauch keine volkswirtschaftlichen Kosten verursacht. 


\section{Nutzungsanreize bei Nicht-Rivalität (Öffentliche Güter, Klubgüter und positive Externalitäten)}

a) Nicht-rivalisierende Güter (öffentliche Güter und Klubgüter). Nicht alle Güter sind rivalisierend. Der Empfang einer Fernsehsendung zum Beispiel wird für einen Zuschauer nicht dadurch gestört, dass ein weiterer Zuschauer die Sendung empfängt. Anders gesagt muss um den zusätzlichen Gebrauch der Fernsehsendung zu ermöglichen keine zusätzliche Einheit des Gutes produziert werden. Technisch gesprochen liegen bei Nicht-Rivalität die Grenzkosten einer zusätzlichen Nutzung bei nul1 ${ }^{99}$. Für sich genommen ist Nicht-Rivalität also eine positive Eigenschaft, die aus ökonomischer Sicht weitgehend ausgenutzt werden sollte. Da Fernsehsendungen ohne Zusatzkosten von weiteren Zuschauern konsumiert werden können, sollten sie aus Effizienzgründen so vielen Zuschauern wie möglich zugänglich gemacht werden.

b) Funktion von Abgaben bei nicht-rivalisierenden Gütern. Im Vergleich zu Nutzungsentgelten für Gemeingüter sollten Preise oder Abgaben bei nicht-rivalisierenden Gütern deshalb die umgekehrte Zielrichtung verfolgen. Ihre Aufgabe ist nicht, den Gebrauch des Gutes (auf ein effizientes Maß) zu beschränken, sondern möglichst keinen Nutzer durch die Abgabenpflicht vom Gebrauch des Gutes abzuhalten. Deshalb ist es problematisch, wenn man für die Nutzung eines nicht-rivalisierenden Gutes Entgelte erhebt - wie beim Pay-per-View im Bezahlfernsehen -, weil dann Nutzungen verhindert werden, die keine Kosten verursachen. Das gilt nun unabhängig davon, ob es sich um ein (reines) öffentliches Gut handelt, bei dem ein Ausschluss nicht möglich ist, oder um ein Klubgut, bei dem Ausschluss und damit Eigentumsrechte möglich sind. Bei letzteren wäre es zwar möglich, Preise zu verlangen und über diese die Produktion des Gutes marktlich zu finanzieren, aber die Entgelte sollten den Gebrauch nicht ineffizient beschränken.

Das gleiche Problem tritt auf, wenn der Staat Klubgüter und öffentliche Güter über Abgaben finanziert ${ }^{100}$. Von Bedeutung ist dann insbesondere, ob die Abgabe - wie bei Gebühren und bestimmten Sonderabgaben - an den Umfang des tatsächlichen Gebrauchs anknüpft, oder ob sie - wie bei Steuern und Beiträgen - unabhängig von der Inanspruchnahme des Gutes erhoben wird. Bei gebrauchsabhängigen Abgaben wird der Abgabenschuldner nämlich seinen Gebrauch auf solche Nutzungen reduzieren, die ihm mehr wert sind als die Abgabe, andere Nutzungen aber unterlassen. Das ist bei rivalisierenden Gütern, insbesondere Gemeingütern, ökonomisch gewollt, bei nicht-rivalisierenden Gütern wie öffentlichen Gütern und Klubgütern aber gerade unerwünscht. Zahlen potentielle Nutzer dagegen Pauschalabgaben, die davon unabhängig sind, in welchem Umfang das Gut tatsächlich in Anspruch genommen wird, hat der Nutzer keinen Anreiz, vom Gebrauch des Gutes abzusehen, solange sein eigener Nutzen positiv ist.

99 Zimmermann/Henke/Broer, (FN 11), S. 53.

100 Die Frage kann sich bei - definitionsgemäß nicht ausschließbaren - öffentlichen Gütern insoweit stellen, als es möglich ist, den Gebrauch zum Tatbestand einer öffentlich-rechtlichen Abgabe zu machen. So war beim Fernsehen vor Erfindung der Verschlüsselungstechnologien zwar kein Ausschluss möglich, aber der Staat konnte den Fernsehkonsum trotzdem zum Gegenstand der hoheitlichen Fernsehgebühr machen. 
Wird beispielsweise zur Finanzierung einer Autobahn ${ }^{101}$ eine kilometerabhängige Maut erhoben, wird man die Autobahn nur benutzen, wenn die Vorteile im Vergleich zur nächstbesten Alternative (Landstraße, öffentliche Verkehrsmittel) höher erscheinen als die Mautgebühr. Werden Bau und Unterhalt einer Autobahn über eine Jahresvignette finanziert, wird man die Vignette erwerben, sobald der voraussichtliche Gesamtvorteil über das Jahr höher ist als der Preis der Vignette. Ab deren Erwerb ist jede Nutzung aber (grenz-)kostenlos, so dass man die Autobahn immer nutzen wird, wenn dies irgendeinen Vorteil, und sei es ein geringer, gegenüber den Alternativen hat (der Vorteil muss also nicht höher sein als die Mautgebühr). Die Vignette wirkt hier also wie eine Flatrate. Wird die Autobahn dagegen über allgemeine Steuern oder über einen Beitrag, etwa für die Halter von $\mathrm{KfZ}$, finanziert, wird man sie auch dann nutzen, wenn die Vorteile übers Jahr den Wert der Vignette nicht erreichen, aber die Nutzung im Einzelfall vorteilhafter erscheint. Sieht man zunächst vom Problem der Überfüllung ab, rivalisiert der Gebrauch von Autobahnen nur in geringem Maße, so dass im Sinne einer effizienten Nutzung eine Steuer gegenüber einer Vignette und die Vignette gegenüber der Kilometermaut vorzuziehen ist. Das gilt umso mehr, wenn die Nutzung des alternativ in Anspruch genommenen Guts, nämlich der Landstraßen, die Anwohner und die Umwelt belastet, also zusätzlich mit negativen Externalitäten verbunden ist. ${ }^{102}$ Durch eine Kilometermaut wird dann nicht nur die Autobahn unternutzt, sondern aufgrund der Ausweichbewegung auch die Landstraße übernutzt. Das gilt nicht für Steuern und nur in geringem Maße für eine Vignette.

\section{Mischgüter und private Güter mit öffentlichen Zwecken}

Viele, wenn nicht die meisten der in der Praxis über nicht-steuerliche Abgaben finanzierten Güter fallen nicht ausschließlich in eine der Güterkategorien, sondern sind auf verschiedene Weisen Mischgüter ${ }^{103}$, vereinigen also mehrere Gütercharakteristika. Zwei in der Praxis häufige Varianten seien angesprochen.

\section{1. Öffentliche Güter mit Kapazitätsgrenzen}

Bei nicht wenigen öffentlichen Gütern und Klubgütern ist Nicht-Rivalität des Gebrauchs nur bis zur Erreichung einer bestimmten Kapazitätsgrenze gegeben: Solange die Autobahn nicht überfüllt ist, beeinträchtigt ein weiteres Kraftfahrzeug das Vorankommen der anderen Verkehrsteilnehmer nur geringfügig. Das gilt aber nicht mehr, wenn bestimmte Kapazitätsgrenzen erreicht sind, etwa im Berufs- oder Ferienverkehr ${ }^{104}$. Dann kann zum Beispiel eine Kombination verschiedener Abgabenarten sinnvoll sein, bei welcher die nicht-rivalisierenden Gebrauchsanteile über pauschale Abgaben finanziert werden, während die rivalisierenden Nutzungen über Nutzungsabgaben mit den von ihnen verursachten (Grenz-)Kosten belastet

$101 \mathrm{Zu}$ den ökonomischen Gütercharakteristika von Straßen s. FN 64.

102 Nash/Shires/Link, Vierteljahreshefte zur Wirtschaftsforschung 79 (2010), S. 13 ff.

103 Gawel, (FN 22), S. 22.

104 Sog. Staugrenzkosten; Gawel/Schmidt, (FN. 9), S. 27 ff.; für eine Schätzung vgl. Nash/Shires/Link, (FN 102), S. 17 ff.). Generell ist perfekte Nicht-Rivalität in der Realität selten gegeben. Auch ohne Staus fallen z.B. Kosten durch Infrastrukturabnutzung und Unfälle an; ebd. S. 20 ff. 
werden $^{105}$. Zum Beispiel könnte man zusätzlich zur Autobahn-Vignette im Berufs- oder Ferienverkehr eine Kilometermaut erheben. Das würde aber je nach der besonderen Konstellation zu einer Reihe mehr oder weniger vorhersehbarer Nebeneffekte führen, die bedacht sein sollten: Die Autofahrer würden etwa im Berufsverkehr in gewissem Umfang auf kostenfreie Landstraßen (oder öffentliche Verkehrsmittel) ausweichen (Substitutionseffekte) ${ }^{106}$, würden insgesamt weniger pendeln, weil sie einen größeren Teil ihres Einkommens für Fahrtkosten aufwenden müssen (Einkommenseffekte), was wiederum verschiedene Pendler je nach Höhe des Einkommens unterschiedlich stark und Pendler stärker als Nicht-Pendler belasten würde (Verteilungseffekte). Es wäre dann eine weitere Aufgabe der ökonomischen Analyse, diese Effekte im Rahmen ihrer Möglichkeit zu prognostizieren ${ }^{107}$, ihre Höhe zu schätzen und Kosten und Nutzen der Maßnahme insgesamt zu vergleichen.

\section{Private Güter mit öffentlichen Zwecken}

Während in der vorigen Kategorie ein Gut seine ökonomische Charakteristik ab einem bestimmten Nutzungsgrad ändert, gibt es auch Güter, die gleichzeitig mehrere Nutzungsdimensionen oder positive Externalitäten aufweisen, die jeweils unterschiedliche Nutzerkreise betreffen und unterschiedliche Gütercharakteristika aufweisen können. Friedhöfe z. B. ermöglichen den Hinterbliebenen eine würdige Bestattung ihres Angehörigen und geben ihnen einen Ort des Totengedenkens. Insoweit sind sie ein privates Gut. Sie dienen aber auch dem Seuchen- und Wasserschutz (öffentliche Güter), werden zudem als Grün- und Erholungsfläche genutzt (insoweit ein Klubgut mit anderen Nutzerkreis), und sind Grundlage einer bestimmten Friedhofskultur (ein öffentliches Gut, primär für die Hinterbliebenen). ${ }^{108}$ Als Abgabenschuldner kommen damit theoretisch mehrere Nutzerkreise in Betracht, in Bezug auf welche die Finanzierung aber je nach Gutscharakteristik (rivalisierend oder nicht-rivalisierend) anders ausgestaltet sein sollten. Der private Nutzungsanteil sollte, wie dies auch geschieht, von den Hinterbliebenen finanziert werden, und zwar wegen der Rivalität des Gebrauchs über Gebühren. Die weiteren öffentlichen Nutzungsanteile (Erholung und öffentliche Gesundheit) sind für die Abgabenschuldner positive Externalitäten, deren Finanzierung nicht ihnen, sondern dem begünstigten Nutzerkreis oder der Allgemeinheit obliegen sollte. Aus ökonomischer Sicht sollte man die Bereitstellungskosten deshalb prozentual um den Anteil der öffentlichen Nutzendimensionen kürzen und letztere über Steuern finanzieren. ${ }^{109}$ Soweit allerdings, wie bei öffentlichen Friedhöfen, ein Benutzungszwang besteht, geht von einer vollen Kostenbelastung kaum Anreizwirkung aus, so dass keine Unterproduktion der öffentlichen Externalitäten droht. Bei Gütern ohne Benutzungszwang kann die volle Kostenbelastung dagegen tendenziell dazu führen, dass das Gut nicht in dem gewünschten Ausmaß in Anspruch genommen

105 Mit zahlreichen Beispielen und Tarifierungsvorschlägen Grossekettler, in Sacksofsky/Wieland (Hrsg.), (FN 3), S. 27 ff. Für andere Gebührenschemata vgl. Hindriks/Myles (FN 20) S. 191 ff.

106 Gawel/Schmidt, (FN 9), S. 212.

107 Für eine Quantifizierung der sozialen Grenzkosten des Straßenverkehrs Nash/Shires/Link, (FN 102).

108 Ausführlich Gawel (FN 21) S. 512 ff.

109 Ebd. 
wird, und damit auch die positiven Externalitäten zu gering ausfallen ${ }^{110}$. Müssten die Eltern die vollen Kosten der Kindergartenbetreuung zahlen, würden weniger Elternteile arbeiten gehen, so dass die erhofften Wirkungen für den Arbeitsmarkt, soweit es sie gibt, geringer ausfielen.

\section{Zusammenfassung}

Die ökonomische Analyse nicht-steuerlicher Abgaben ist eine Korrespondenzbetrachtung von Gutscharakteristik und adäquaten Finanzierungsinstrumenten (Gawel). Bei fehlender oder ineffizienter Ausschließbarkeit vom Gebrauch ist danach eine über nicht-steuerliche Abgaben finanzierte Produktion von Gütern in Betracht zu ziehen, und zwar für Öffentliche Güter, Klubgüter und für private Güter mit positiven Externalitäten, meritorischen Nutzendimensionen oder Verteilungszwecken. Die zur Finanzierung dieser Güter erhobenen nichtsteuerlichen Abgaben sollten dabei im Hinblick auf eine effiziente Nutzung ausgewählt und ausgestaltet werden. Bei nicht-rivalisierenden Nutzungsdimensionen sollten Anreize vermieden werden, die die Nutzung des Gutes beschränken, während sie bei rivalisierendem Gebrauch umgekehrt auf eine angemessene Beschränkung der Nutzung ausgerichtet sein sollten.

Staatsaufgaben können finanzwissenschaftlich als staatliche Produktion von Gütern gedeutet werden. Auf dieser Grundlage lassen sich die verfassungsrechtlichen Anforderungen an die Rechtfertigung nicht-steuerlicher Abgaben in den Kategorien der Kollektivgüterlehre rekonstruieren. Während die Finanzwissenschaften, wie oben skizziert, die Verknüpfung von Finanzierung und Lenkungswirkungen untersuchen, begreift das Verfassungsrecht die Unterscheidung von Finanzierungs- und Lenkungszwecken auf einer anderen Ebene, die sich finanzwissenschaftlich als Unterscheidung von positiven und negativen Kollektivgütern erweist. Finanzierungszwecke können dabei die Erhebung nicht-steuerlicher Abgaben nur rechtfertigen, wenn sie der Produktion von kollektiven Gütern dienen - öffentlichen Gütern, Klubgütern oder privaten Gütern mit öffentlichen Zwecken -, die den Abgabenschuldnern individuell, der Möglichkeit nach oder als homogene Gruppe zugute kommen. Eine zwangsweise Bereitstellung gruppennütziger öffentlicher Güter setzt außerdem voraus, dass der Nutzen evident ist und die Betroffenen das Gut nicht selbst produzieren können. Lenkungszwecke (im verfassungsrechtlichen Sinn) beziehen sich dagegen auf die Verantwortlichkeit einzelner Bürger oder Unternehmen, oder einer insoweit homogenen Gruppe, für negative Externalitäten, insbesondere für die Übernutzung von Gemeingütern. Aus Äquivalenzgründen sollten Lenkungszwecke aber nur als Nebenwirkung der Abschöpfung eines Vorteils aus der Ressourcennutzung zulässig sein, darüber hinaus aber Lenkungssteuern vorbehalten bleiben. 